\title{
Finite energy electroweak dyon
}

\author{
Kyoungtae Kimm ${ }^{1}$, J. H. Yoon ${ }^{2}$, Y. M. Cho ${ }^{3,4, a}$ \\ ${ }^{1}$ Faculty of Liberal Education, Seoul National University, Seoul 151-747, Korea \\ 2 Department of Physics, College of Natural Sciences, Konkuk University, Seoul 143-701, Korea \\ ${ }^{3}$ Administration Building 310-4, Konkuk University, Seoul 143-701, Korea \\ ${ }^{4}$ School of Physics and Astronomy, Seoul National University, Seoul 151-742, Korea
}

Received: 28 October 2014 / Accepted: 26 January 2015 / Published online: 10 February 2015

(C) The Author(s) 2015. This article is published with open access at Springerlink.com

\begin{abstract}
The latest MoEDAL experiment at LHC to detect the electroweak monopole makes the theoretical prediction of the monopole mass an urgent issue. We discuss three different ways to estimate the mass of the electroweak monopole. We first present the dimensional and scaling arguments which indicate the monopole mass to be around 4 to $10 \mathrm{TeV}$. To justify this we construct finite energy analytic dyon solutions which could be viewed as the regularized Cho-Maison dyon, modifying the coupling strength at short distance. Our result demonstrates that a genuine electroweak monopole whose mass scale is much smaller than the grand unification scale can exist, which can actually be detected at the present LHC.
\end{abstract}

\section{Introduction}

The recent discover of the Higgs particle at LHC and Tevatron has reconfirmed that the electroweak theory of Weinberg and Salam provides the true unification of electromagnetic and weak interactions [1-3]. Indeed the discovery of the Higgs particle has been claimed to be the "final" test of the standard model. This, however, might be a premature claim. The real final test should come from the discovery of the electroweak monopole, because the standard model predicts it [4-7]. In fact the existence of the monopole topology in the standard model tells that the discovery of the monopole must be the topological test of the standard model.

In this sense it is timely that the latest MoEDAL detector ("The Magnificent Seventh") at LHC is actively searching for such monopole [8-11]. To detect the electroweak monopole experimentally, however, it is important to estimate the monopole mass in advance. The purpose of this paper is to estimate the mass of the electroweak monopole. We show that the monopole mass is expected to be around 4 to $7 \mathrm{TeV}$.

\footnotetext{
a e-mail: ymcho7@konkuk.ac.kr
}

Ever since Dirac [12] has introduced the concept of the magnetic monopole, the monopoles have remained a fascinating subject. The Abelian monopole has been generalized to the non-Abelian monopoles by $\mathrm{Wu}$ and Yang [1316] who showed that the pure $S U$ (2) gauge theory allows a point-like monopole, and by 't Hooft and Polyakov [17-20] who have constructed a finite energy monopole solution in Georgi-Glashow model as a topological soliton. Moreover, the monopole in grand unification has been constructed by Dokos and Tomaras [21].

In the interesting case of the electroweak theory of Weinberg and Salam, however, it has been asserted that there exists no topological monopole of physical interest $[22,23]$. The basis for this "non-existence theorem" is, of course, that with the spontaneous symmetry breaking the quotient space $S U(2) \times U(1)_{Y} / U(1)_{\text {em }}$ allows no non-trivial second homotopy. This has led many people to believe that there is no monopole in Weinberg-Salam model.

This claim, however, has been shown to be false. If the electroweak unification of Weinberg and Salam is correct, the standard model must have a monopole which generalizes the Dirac monopole. Moreover, it has been shown that the standard model has a new type of monopole and dyon solutions $[4,5]$. This was based on the observation that the Weinberg-Salam model, with the $U(1)_{Y}$, could be viewed as a gauged $C P^{1}$ model in which the (normalized) Higgs doublet plays the role of the $C P^{1}$ field. So the Weinberg-Salam model does have exactly the same non-trivial second homotopy as the Georgi-Glashow model which allows topological monopoles.

Once this is understood, one could proceed to construct the desired monopole and dyon solutions in the Weinberg-Salam model. Originally the electroweak monopole and dyon solutions were obtained by numerical integration. But a mathematically rigorous existence proof has been established which endorses the numerical results, and the solutions are now referred to as Cho-Maison monopole and dyon $[6,7]$. 
It should be emphasized that the Cho-Maison monopole is completely different from the "electroweak monopole" derived from the Nambu electroweak string. In his continued search for the string-like objects in physics, Nambu has demonstrated the existence of a rotating dumb bell made of the monopole anti-monopole pair connected by the neutral string of $Z$-boson flux (actually the $S U$ (2) flux) in WeinbergSalam model $[24,25]$. Taking advantage of Nambu's pioneering work, others claimed to have discovered another type of electroweak monopole, simply by making the string infinitely long and moving the anti-monopole to infinity [26]. This "electroweak monopole", however, must carry a fractional magnetic charge and cannot be isolated with finite energy. Moreover, this has no spherical symmetry which is manifest in the Cho-Maison monopole [4,5].

The existence of the electroweak monopole makes the experimental confirmation of the monopole an urgent issue. Till recently the experimental effort for the monopole detection has been on the Dirac monopole [27]. But the electroweak unification of Maxwell's theory requires the modification of the Dirac monopole, and this modification changes the Dirac monopole to the Cho-Maison monopole. This means that the monopole which should exist in the real world is not likely to be the Dirac monopole but the electroweak monopole.

To detect the electroweak monopole experimentally, it is important to estimate the mass of the monopole theoretically. Unfortunately the Cho-Maison monopole carries an infinite energy at the classical level, so that the monopole mass is not determined. This is because it can be viewed as a hybrid between the Dirac monopole and the 't HooftPolyakov monopole, so that it has a $U(1)_{\mathrm{em}}$ point singularity at the center even though the $S U(2)$ part is completely regular.

A priori there is nothing wrong with this. Classically the electron has an infinite electric energy but a finite mass. But for the experimental search for the monopole we need a solid idea about the monopole mass. In this paper we show how to predict the mass of the electroweak monopole. Based on the dimensional argument we first show that the monopole mass should be of the order of $1 / \alpha$ times the W-boson mass, or around $10 \mathrm{TeV}$. To back up this we adopt the scaling argument to predict the mass to be around $4 \mathrm{TeV}$. Finally, we show how the quantum correction could regularize the point singularity of the Cho-Maison dyon, and construct finite energy electroweak dyon solutions introducing the effective action of the standard model. Our result suggests that the electroweak monopole with the mass around 4 to $7 \mathrm{TeV}$ could exist, which implies that there is a very good chance that the MoEDAL at the present $\mathrm{LHC}$ can detect the electroweak monopole.

The paper is organized as follows. In Sect. 2 we review the Cho-Maison dyon for later purpose. In Sect. 3 we provide two arguments, the dimensional and scaling arguments, which indicate that the mass of the electroweak monopole could be around 4 to $10 \mathrm{TeV}$. In Sect. 4 we discuss the Abelian decomposition and gauge independent Abelianization of Weinberg-Salam model and Georgi-Glashow model to help us how to regularize the Cho-Maison monopole. In Sect. 5 we discuss two different methods to regularize the Cho-Maison dyon with the quantum correction which modifies the coupling constants at short distance, and construct finite energy dyon solutions which support the scaling argument. In Sect. 6 we discuss another way to make the Cho-Maison dyon regular, by enlarging the gauge group $S U(2) \times U(1)_{Y}$ to $S U(2) \times S U(2)_{Y}$. Finally in Sect. 7 we discuss the physical implications of our results.

\section{Cho-Maison dyon in Weinberg-Salam model: a review}

Before we construct a finite energy dyon solution in the electroweak theory we must understand how one can obtain the infinite energy Cho-Maison dyon solution first. Let us start with the Lagrangian which describes (the bosonic sector of) the Weinberg-Salam theory

$$
\begin{aligned}
\mathcal{L} & =-\left|\mathcal{D}_{\mu} \phi\right|^{2}-\frac{\lambda}{2}\left(\phi^{\dagger} \phi-\frac{\mu^{2}}{\lambda}\right)^{2}-\frac{1}{4} \vec{F}_{\mu \nu}^{2}-\frac{1}{4} G_{\mu \nu}^{2} \\
\mathcal{D}_{\mu} \phi & =\left(\partial_{\mu}-i \frac{g}{2} \vec{\tau} \cdot \vec{A}_{\mu}-i \frac{g^{\prime}}{2} B_{\mu}\right) \phi \\
& =\left(D_{\mu}-i \frac{g^{\prime}}{2} B_{\mu}\right) \phi
\end{aligned}
$$

where $\phi$ is the Higgs doublet, $\vec{F}_{\mu \nu}$ and $G_{\mu \nu}$ are the gauge field strengths of $S U(2)$ and $U(1)_{Y}$ with the potentials $\vec{A}_{\mu}$ and $B_{\mu}$, and $g$ and $g^{\prime}$ are the corresponding coupling constants. Notice that $D_{\mu}$ describes the covariant derivative of the $S U(2)$ subgroup only. With

$\phi=\frac{1}{\sqrt{2}} \rho \xi, \quad\left(\xi^{\dagger} \xi=1\right)$,

where $\rho$ and $\xi$ are the Higgs field and unit doublet, we have

$$
\begin{aligned}
\mathcal{L}= & -\frac{1}{2}\left(\partial_{\mu} \rho\right)^{2}-\frac{\rho^{2}}{2}\left|\mathcal{D}_{\mu} \xi\right|^{2}-\frac{\lambda}{8}\left(\rho^{2}-\frac{2 \mu^{2}}{\lambda}\right)^{2} \\
& -\frac{1}{4} \vec{F}_{\mu \nu}^{2}-\frac{1}{4} G_{\mu \nu}^{2} .
\end{aligned}
$$

Notice that the $U(1)_{Y}$ coupling of $\xi$ makes the theory a gauge theory of $C P^{1}$ field $[4,5]$.

From (1) one has the following equations of motion:

$$
\begin{aligned}
\partial^{2} \rho & =\left|\mathcal{D}_{\mu} \xi\right|^{2} \rho+\frac{\lambda}{2}\left(\rho^{2}-\frac{2 \mu^{2}}{\lambda}\right) \rho, \\
\mathcal{D}^{2} \xi & =-2 \frac{\partial_{\mu} \rho}{\rho} \mathcal{D}_{\mu} \xi+\left[\xi^{\dagger} \mathcal{D}^{2} \xi+2 \frac{\partial_{\mu} \rho}{\rho}\left(\xi^{\dagger} \mathcal{D}_{\mu} \xi\right)\right] \xi,
\end{aligned}
$$


$D_{\mu} \vec{F}_{\mu \nu}=i \frac{g}{2} \rho^{2}\left[\xi^{\dagger} \vec{\tau}\left(\mathcal{D}_{\nu} \xi\right)-\left(\mathcal{D}_{\nu} \xi\right)^{\dagger} \vec{\tau} \xi\right]$,

$\partial_{\mu} G_{\mu \nu}=i \frac{g^{\prime}}{2} \rho^{2}\left[\xi^{\dagger}\left(\mathcal{D}_{\nu} \xi\right)-\left(\mathcal{D}_{\nu} \xi\right)^{\dagger} \xi\right]$.

Now we choose the following ansatz in the spherical coordinates $(t, r, \theta, \varphi)$ :

$$
\begin{aligned}
\rho & =\rho(r), \quad \xi=i\left(\begin{array}{c}
\sin (\theta / 2) e^{-i \varphi} \\
-\cos (\theta / 2)
\end{array}\right), \\
\vec{A}_{\mu} & =\frac{1}{g} A(r) \partial_{\mu} t \hat{r}+\frac{1}{g}(f(r)-1) \hat{r} \times \partial_{\mu} \hat{r}, \\
B_{\mu} & =\frac{1}{g^{\prime}} B(r) \partial_{\mu} t-\frac{1}{g^{\prime}}(1-\cos \theta) \partial_{\mu} \varphi .
\end{aligned}
$$

Notice that $\xi^{\dagger} \vec{\tau} \xi=-\hat{r}$. Moreover, $\vec{A}_{\mu}$ describes the $\mathrm{Wu}-$ Yang monopole when $A(r)=f(r)=0$. So the ansatz is spherically symmetric. Of course, $\xi$ and $B_{\mu}$ have an apparent string singularity along the negative $z$-axis, but this singularity is a pure gauge artifact which can easily be removed making the $U(1)_{Y}$ bundle non-trivial. So the above ansatz describes a most general spherically symmetric ansatz of an electroweak dyon.

Here we emphasize the importance of the non-trivial nature of $U(1)_{Y}$ gauge symmetry to make the ansatz spherically symmetric. Without the extra $U(1)_{Y}$ the Higgs doublet does not allow a spherically symmetric ansatz. This is because the spherical symmetry for the gauge field involves the embedding of the radial isotropy group $S O(2)$ into the gauge group that requires the Higgs field to be invariant under the $U(1)$ subgroup of $S U(2)$. This is possible with a Higgs triplet, but not with a Higgs doublet [28]. In fact, in the absence of the $U(1)_{Y}$ degrees of freedom, the above ansatz describes the $S U(2)$ sphaleron which is not spherically symmetric [29-31].

To see this, one might try to remove the string in $\xi$ with the $U(1)$ subgroup of $S U(2)$. But this $U(1)$ will necessarily change $\hat{r}$ and thus violate the spherical symmetry. This means that there is no $S U(2)$ gauge transformation which can remove the string in $\xi$ and at the same time keeps the spherical symmetry intact. The situation changes with the inclusion of the $U(1)_{Y}$ in the standard model, which naturally makes $\xi$ a $C P^{1}$ field $[4,5]$. This allows the spherical symmetry for the Higgs doublet.

To understand the physical content of the ansatz we perform the following gauge transformation on (5):

$$
\begin{aligned}
& \xi \rightarrow U \xi=\left(\begin{array}{l}
0 \\
1
\end{array}\right), \\
& U=i\left(\begin{array}{cc}
\cos (\theta / 2) & \sin (\theta / 2) e^{-i \varphi} \\
-\sin (\theta / 2) e^{i \varphi} & \cos (\theta / 2)
\end{array}\right),
\end{aligned}
$$

and find that in this unitary gauge we have

$\hat{r} \rightarrow\left(\begin{array}{l}0 \\ 0 \\ 1\end{array}\right)$

$\vec{A}_{\mu} \rightarrow \frac{1}{g}\left(\begin{array}{c}-f(r)\left(\sin \varphi \partial_{\mu} \theta+\sin \theta \cos \varphi \partial_{\mu} \varphi\right) \\ f(r)\left(\cos \varphi \partial_{\mu} \theta-\sin \theta \sin \varphi \partial_{\mu} \varphi\right) \\ A(r) \partial_{\mu} t-(1-\cos \theta) \partial_{\mu} \varphi\end{array}\right)$.

So introducing the electromagnetic and neutral $Z$-boson potentials $A_{\mu}^{(\mathrm{em})}$ and $Z_{\mu}$ with the Weinberg angle $\theta_{\mathrm{w}}$

$$
\begin{aligned}
\left(\begin{array}{c}
A_{\mu}^{(\mathrm{em})} \\
Z_{\mu}
\end{array}\right) & =\left(\begin{array}{cc}
\cos \theta_{\mathrm{w}} & \sin \theta_{\mathrm{w}} \\
-\sin \theta_{\mathrm{w}} & \cos \theta_{\mathrm{w}}
\end{array}\right)\left(\begin{array}{c}
B_{\mu} \\
A_{\mu}^{3}
\end{array}\right) \\
& =\frac{1}{\sqrt{g^{2}+g^{\prime 2}}}\left(\begin{array}{cc}
g & g^{\prime} \\
-g^{\prime} & g
\end{array}\right)\left(\begin{array}{c}
B_{\mu} \\
A_{\mu}^{3}
\end{array}\right),
\end{aligned}
$$

we can express the ansatz (5) in terms of the physical fields

$$
\begin{aligned}
W_{\mu}= & \frac{1}{\sqrt{2}}\left(A_{\mu}^{1}+i A_{\mu}^{2}\right)=\frac{i}{g} \frac{f(r)}{\sqrt{2}} e^{i \varphi}\left(\partial_{\mu} \theta+i \sin \theta \partial_{\mu} \varphi\right), \\
A_{\mu}^{(\mathrm{em})}= & e\left(\frac{1}{g^{2}} A(r)+\frac{1}{g^{\prime 2}} B(r)\right) \partial_{\mu} t \\
& -\frac{1}{e}(1-\cos \theta) \partial_{\mu} \varphi, \\
Z_{\mu}= & \frac{e}{g g^{\prime}}(A(r)-B(r)) \partial_{\mu} t,
\end{aligned}
$$

where $W_{\mu}$ is the $W$-boson and $e$ is the electric charge

$e=\frac{g g^{\prime}}{\sqrt{g^{2}+g^{\prime 2}}}=g \sin \theta_{\mathrm{w}}=g^{\prime} \cos \theta_{\mathrm{w}}$.

This clearly shows that the ansatz is for the electroweak dyon.

The spherically symmetric ansatz reduces the equations of motion to

$$
\begin{aligned}
\ddot{\rho}+\frac{2}{r} \dot{\rho}-\frac{f^{2}}{2 r^{2}} \rho & =-\frac{1}{4}(A-B)^{2} \rho+\frac{\lambda}{2}\left(\rho^{2}-\frac{2 \mu^{2}}{\lambda}\right) \rho, \\
\ddot{f}-\frac{f^{2}-1}{r^{2}} f & =\left(\frac{g^{2}}{4} \rho^{2}-A^{2}\right) f, \\
\ddot{A}+\frac{2}{r} \dot{A}-\frac{2 f^{2}}{r^{2}} A & =\frac{g^{2}}{4} \rho^{2}(A-B), \\
\ddot{B}+\frac{2}{r} \dot{B} & =-\frac{g^{\prime 2}}{4} \rho^{2}(A-B) .
\end{aligned}
$$

Obviously this has a trivial solution

$\rho=\rho_{0}=\sqrt{2 \mu^{2} / \lambda}, \quad f=0, \quad A=B=0$,

which describes the point monopole in Weinberg-Salam model

$A_{\mu}^{(\mathrm{em})}=-\frac{1}{e}(1-\cos \theta) \partial_{\mu} \varphi$.

This monopole has two remarkable features. First, this is the electroweak generalization of the Dirac monopole, but not the Dirac monopole. It has the electric charge $4 \pi / e$, not 
$2 \pi / e[4,5]$. Second, this monopole naturally admits a nontrivial dressing of weak bosons. Indeed, with the non-trivial dressing, the monopole becomes the Cho-Maison dyon.

To see this let us choose the following boundary condition:

$\rho(0)=0, \quad f(0)=1, \quad A(0)=0, \quad B(0)=b_{0}$,

$\rho(\infty)=\rho_{0}, \quad f(\infty)=0, \quad A(\infty)=B(\infty)=A_{0}$.

Then we can show that the Eq. (10) admits a family of solutions labeled by the real parameter $A_{0}$ lying in the range [4-7]

$0 \leq A_{0}<\min \left(e \rho_{0}, \frac{g}{2} \rho_{0}\right)$.

In this case all four functions $f(r), \rho(r), A(r)$, and $B(r)$ must be positive for $r>0$, and $A(r) / g^{2}+B(r) / g^{2}$ and $B(r)$ become increasing functions of $r$. So we have $0 \leq b_{0} \leq$ $A_{0}$. Furthermore, we have $B(r) \geq A(r) \geq 0$ for all range, and $B(r)$ must approach $A(r)$ with an exponential damping. Notice that, with the experimental fact $\sin ^{2} \theta_{\mathrm{w}}=0.2312$, (14) can be written as $0 \leq A_{0}<e \rho_{0}$.

With the boundary condition (13) we can integrate (10). For example, with $A=B=0$, we have the Cho-Maison monopole. In general, with $A_{0} \neq 0$, we find the Cho-Maison dyon $[4,5]$.

Near the origin the dyon solution has the following behavior:

$\rho \simeq \alpha_{1} r^{\delta_{-}}, \quad f \simeq 1+\beta_{1} r^{2}$,

$A \simeq a_{1} r, \quad B \simeq b_{0}+b_{1} r^{2 \delta_{+}}$,

where $\delta_{ \pm}=(\sqrt{3} \pm 1) / 2$. Asymptotically it has the following behavior:

$\rho \simeq \rho_{0}+\rho_{1} \frac{\exp (-\sqrt{2} \mu r)}{r}, \quad f \simeq f_{1} \exp (-\omega r)$,

$A \simeq A_{0}+\frac{A_{1}}{r}, \quad B \simeq A+B_{1} \frac{\exp (-v r)}{r}$,

where $\omega=\sqrt{\left(g \rho_{0}\right)^{2} / 4-A_{0}^{2}}$, and $v=\sqrt{\left(g^{2}+g^{\prime 2}\right)} \rho_{0} / 2$. The physical meaning of the asymptotic behavior must be clear. Obviously $\rho, f$, and $A-B$ represent the Higgs boson, $W$-boson, and $Z$-boson whose masses are given by $M_{H}=$ $\sqrt{2} \mu=\sqrt{\lambda} \rho_{0}, M_{W}=g \rho_{0} / 2$, and $M_{Z}=\sqrt{g^{2}+g^{\prime 2}} \rho_{0} / 2$.

So (16) tells that $M_{H}, \sqrt{1-\left(A_{0} / M_{W}\right)^{2}} M_{W}$, and $M_{Z}$ determine the exponential damping of the Higgs boson, $W$-boson, and $Z$-boson to their vacuum expectation values asymptotically. Notice that it is $\sqrt{1-\left(A_{0} / M_{W}\right)^{2}} M_{W}$, but not $M_{W}$, which determines the exponential damping of the $W$-boson. This tells that the electric potential of the dyon slows down the exponential damping of the $W$-boson, which is reasonable.
The dyon has the following electromagnetic charges:

$$
\begin{aligned}
q_{e} & =-\frac{8 \pi}{e} \sin ^{2} \theta_{\mathrm{w}} \int_{0}^{\infty} f^{2} A \mathrm{~d} r=\frac{4 \pi}{e} A_{1}, \\
q_{m} & =\frac{4 \pi}{e} .
\end{aligned}
$$

Also, the asymptotic condition (16) assures that the dyon does not carry any neutral charge,

$Z_{e}=-\left.\frac{4 \pi e}{g g^{\prime}}\left[r^{2}(\dot{A}-\dot{B})\right]\right|_{r=\infty}=0$,

$Z_{m}=0$.

Furthermore, notice that the dyon equation (10) is invariant under the reflection

$A \rightarrow-A, \quad B \rightarrow-B$.

This means that, for a given magnetic charge, there are always two dyon solutions which carry opposite electric charges $\pm q_{e}$. Clearly the signature of the electric charge of the dyon is determined by the signature of the boundary value $A_{0}$.

We can also have the anti-monopole or in general antidyon solution, the charge conjugate state of the dyon, which has the magnetic charge $q_{m}=-4 \pi / e$ with the following ansatz:

$$
\begin{aligned}
\rho^{\prime} & =\rho(r), \quad \xi^{\prime}=-i\left(\begin{array}{c}
\sin (\theta / 2) e^{+i \varphi} \\
-\cos (\theta / 2)
\end{array}\right), \\
\vec{A}_{\mu}^{\prime} & =-\frac{1}{g} A(r) \partial_{\mu} t \hat{r}^{\prime}+\frac{1}{g}(f(r)-1) \hat{r}^{\prime} \times \partial_{\mu} \hat{r}^{\prime}, \\
B_{\mu}^{\prime} & =-\frac{1}{g^{\prime}} B(r) \partial_{\mu} t+\frac{1}{g^{\prime}}(1-\cos \theta) \partial_{\mu} \varphi, \\
\hat{r}^{\prime} & =-\xi^{\prime \dagger} \vec{\tau} \xi^{\prime}=(\sin \theta \cos \phi,-\sin \theta \sin \phi, \cos \theta) .
\end{aligned}
$$

Notice that the ansatz is basically the complex conjugation of the dyon ansatz.

To understand the meaning of the anti-dyon ansatz notice that in the unitary gauge

$\xi^{\prime} \rightarrow U^{\prime} \xi^{\prime}=\left(\begin{array}{l}0 \\ 1\end{array}\right)$,

$U^{\prime}=-i\left(\begin{array}{cc}\cos (\theta / 2) & \sin (\theta / 2) e^{i \varphi} \\ -\sin (\theta / 2) e^{-i \varphi} & \cos (\theta / 2)\end{array}\right)$,

we have

$\vec{A}_{\mu}^{\prime} \rightarrow \frac{1}{g}\left(\begin{array}{c}f(r)\left(\sin \varphi \partial_{\mu} \theta+\sin \theta \cos \varphi \partial_{\mu} \varphi\right) \\ f(r)\left(\cos \varphi \partial_{\mu} \theta-\sin \theta \sin \varphi \partial_{\mu} \varphi\right) \\ -A(r) \partial_{\mu} t+(1-\cos \theta) \partial_{\mu} \varphi\end{array}\right)$.

So in terms of the physical fields the ansatz (20) is expressed by

$$
\begin{aligned}
W_{\mu}^{\prime} & =\frac{i}{g} \frac{f(r)}{\sqrt{2}} e^{-i \varphi}\left(\partial_{\mu} \theta-i \sin \theta \partial_{\mu} \varphi\right)=-W_{\mu}^{*}, \\
A_{\mu}^{(\mathrm{em})} & =-e\left(\frac{1}{g^{2}} A(r)+\frac{1}{g^{\prime 2}} B(r)\right) \partial_{\mu} t
\end{aligned}
$$




$$
\begin{gathered}
+\frac{1}{e}(1-\cos \theta) \partial_{\mu} \varphi, \\
Z_{\mu}^{\prime}=-\frac{e}{g g^{\prime}}(A(r)-B(r)) \partial_{\mu} t=-Z_{\mu} .
\end{gathered}
$$

This clearly shows that the electric and magnetic charges of the ansatz (20) are the opposite of the dyon ansatz, which confirms that the ansatz indeed describes the anti-dyon.

With the ansatz (20) we have exactly the same Eq. (10) for the anti-dyon. This assures that the standard model has the anti-dyon as well as the dyon.

The above discussion tells that the $\mathrm{W}$ and $\mathrm{Z}$ boson part of the anti-dyon solution is basically the complex conjugation of the dyon solution. This, of course, is natural from the physical point of view. On the other hand there is one minor point to be clarified here. Since the topological charge of the monopole is given by the second homotopy defined by $\hat{r}=-\xi^{\dagger} \vec{\tau} \xi$, one might expect that $\hat{r}^{\prime}$ defined by the anti-dyon ansatz $\xi^{\prime}=\xi^{*}$ must be $-\hat{r}$. But this is not so, and we have to explain why.

To understand this notice that we can change $\hat{r}^{\prime}$ to $-\hat{r}$ by a SU(2) gauge transformation, by the $\pi$-rotation along the $y$ axis. With this gauge transformation the ansatz (20) changes to

$$
\begin{aligned}
& \xi^{\prime} \rightarrow i\left(\begin{array}{c}
\cos (\theta / 2) \\
\sin (\theta / 2) e^{+i \varphi}
\end{array}\right), \quad \hat{r}^{\prime} \rightarrow-\hat{r}, \\
& \vec{A}_{\mu} \rightarrow-\frac{1}{g} A(r) \partial_{\mu} t \hat{r}+\frac{1}{g}(f(r)-1) \hat{r} \times \partial_{\mu} \hat{r} .
\end{aligned}
$$

This tells that the monopole topology defined by $\hat{r}^{\prime}$ is the same as that of $\hat{r}$.

Since the Cho-Maison solution is obtained numerically, one might like to have a mathematically rigorous existence proof of the Cho-Maison dyon. The existence proof is nontrivial, because the equation of motion (10) is not the EulerLagrange equation of the positive definite energy (26), but that of the indefinite action

$$
\begin{aligned}
\mathcal{L}= & -4 \pi \int_{0}^{\infty} \mathrm{d} r\left\{\frac{1}{2}(r \dot{\rho})^{2}+\frac{\lambda r^{2}}{8}\left(\rho^{2}-\rho_{0}^{2}\right)^{2}\right. \\
& +\frac{1}{4} f^{2} \rho^{2}+\frac{1}{g^{2}}\left(\dot{f}^{2}-\frac{1}{2}(r \dot{A})^{2}-f^{2} A^{2}\right)-\frac{1}{2 g^{\prime 2}}(r \dot{B})^{2} \\
& \left.-\frac{r^{2}}{8}(B-A)^{2} \rho^{2}+\frac{1}{2 r^{2}}\left(\frac{1}{g^{\prime 2}}+\frac{1}{g^{2}}\left(f^{2}-1\right)^{2}\right)\right\} .
\end{aligned}
$$

Fortunately the existence proof has been established by Yang $[6,7]$.

Before we leave this section it is worth to re-address the important question again: Does the standard model predict the monopole? Notice that the Dirac monopole in electrodynamics is optional: It can exist only when $U(1)_{\mathrm{em}}$ is nontrivial, but there is no reason why this has to be so. If so, why cannot the electroweak monopole be optional?
As we have pointed out, the non-trivial $U(1)_{Y}$ is crucial for the existence of the monopole in the standard model. So the question here is why the $U(1)_{Y}$ must be non-trivial. To see why, notice that in the standard model $U(1)_{\mathrm{em}}$ comes from two $U(1)$, the $U(1)$ subgroup of $S U(2)$ and $U(1)_{Y}$, and it is well known that the $U(1)$ subgroup of $S U(2)$ is non-trivial. Now, to obtain the electroweak monopole we have to make the linear combination of two monopoles; that of the $U(1)$ subgroup of $S U(2)$ and $U(1)_{Y}$. This must be clear from (8).

In this case the mathematical consistency requires the two potentials $A_{\mu}^{3}$ and $B_{\mu}$ (and two $U(1)$ ) to have the same structure, in particular the same topology. But we already know that $A_{\mu}^{3}$ is non-trivial. So $B_{\mu}$, and the corresponding $U(1)_{Y}$, has to be non-trivial. In other words, requiring $U(1)_{Y}$ to be trivial is inconsistent (i.e., in contradiction with the selfconsistency) in the standard model. This tells that, unlike Maxwell's theory, the $U(1)_{\mathrm{em}}$ in the standard model must be non-trivial. This assures that the standard model must have the monopole.

But ultimately this question has to be answered by the experiment. So the discovery of the monopole must be the topological test of the standard model, which has never been done before. This is why MoEDAL is so important.

\section{Mass of the electroweak monopole}

To detect the electroweak monopole experimentally, we have to have a firm idea on the mass of the monopole. Unfortunately, at the classical level we cannot estimate the mass of the Cho-Maison monopole, because it has a point singularity at the center which makes the total energy infinite.

Indeed the ansatz (5) gives the following energy:

$$
\begin{aligned}
E= & E_{0}+E_{1}, \\
E_{0}= & 4 \pi \int_{0}^{\infty} \frac{\mathrm{d} r}{2 r^{2}}\left\{\frac{1}{g^{\prime 2}}+\frac{1}{g^{2}}\left(f^{2}-1\right)^{2}\right\}, \\
E_{1}= & 4 \pi \int_{0}^{\infty} \mathrm{d} r\left\{\frac{1}{2}(r \dot{\rho})^{2}+\frac{1}{g^{2}}\left(\dot{f}^{2}+\frac{1}{2}(r \dot{A})^{2}\right.\right. \\
& \left.+f^{2} A^{2}\right)+\frac{1}{2 g^{\prime 2}}(r \dot{B})^{2}+\frac{\lambda r^{2}}{8}\left(\rho^{2}-\rho_{0}^{2}\right)^{2} \\
& \left.+\frac{1}{4} f^{2} \rho^{2}+\frac{r^{2}}{8}(B-A)^{2} \rho^{2}\right\} .
\end{aligned}
$$

The boundary condition (13) guarantees that $E_{1}$ is finite. As for $E_{0}$ we can minimize it with the boundary condition $f(0)=1$, but even with this $E_{0}$ becomes infinite. Of course the origin of this infinite energy is obvious, which is precisely due to the magnetic singularity of $B_{\mu}$ at the origin. This means that one cannot predict the mass of the dyon. Physically it remains arbitrary.

To estimate the monopole mass theoretically, we have to regularize the point singularity of the Cho-Maison dyon. One 
might try to do that introducing the gravitational interaction, in which case the mass is fixed by the asymptotic behavior of the gravitational potential. But the magnetic charge of the monopole is not likely to change the character of the singularity, so that asymptotically the leading order of the gravitational potential becomes that of the Reissner-Nordstrom type [32-35]. This implies the gravitational interaction may not help us to estimate the monopole mass.

To make the energy of the Cho-Maison monopole finite, notice that the origin of the infinite energy is the first term $1 / g^{\prime 2}$ in $E_{0}$ in (26). A simple way to make this term finite is to introduce a UV-cutoff which removes this divergence. This type of cutoff could naturally come from the quantum correction of the coupling constants. In fact, since the quantum correction changes $g^{\prime}$ to the running coupling $\bar{g}^{\prime}, E_{0}$ can become finite if $\bar{g}^{\prime}$ diverges at short distance.

We will discuss how such quantum correction could take place later, but before doing that we present two arguments, the dimensional argument and the scaling argument, which could give us a rough estimate of the monopole mass.

\subsection{Dimensional argument}

To have the order estimate of the monopole mass it is important to realize that, roughly speaking, the monopole mass comes from the Higgs mechanism which generates the mass to the W-boson. This can easily be seen for the 't HooftPolyakov monopole in the Georgi-Glashow model:

$\mathcal{L}_{G G}=-\frac{1}{4} \vec{F}_{\mu \nu}^{2}-\frac{1}{2}\left(D_{\mu} \vec{\Phi}\right)^{2}-\frac{\lambda}{4}\left(\vec{\Phi}^{2}-\frac{\mu^{2}}{\lambda}\right)^{2}$,

where $\vec{\Phi}$ is the Higgs triplet. Here the monopole ansatz is given by

$\vec{\Phi}=\rho \hat{r}, \quad \vec{A}_{\mu}=\vec{C}_{\mu}+\vec{W}_{\mu}$,

$\vec{C}_{\mu}=-\frac{1}{g} \hat{r} \times \partial_{\mu} \hat{r}, \quad \vec{W}_{\mu}=-f \vec{C}_{\mu}$,

where $\vec{C}_{\mu}$ represents the $\mathrm{Wu}-$ Yang monopole potential [13, $14,36]$. Notice that the $\mathrm{W}$-boson part of the monopole is given by the Wu-Yang potential, except for the overall amplitude $f$.

With this we clearly have

$\left|D_{\mu} \Phi\right|^{2}=\left(\partial_{\mu} \rho\right)^{2}+g^{2} \rho^{2} f^{2}\left(\vec{C}_{\mu}\right)^{2}$.

So, when the Higgs field has a non-vanishing vacuum expectation value, $\vec{C}_{\mu}$ acquires a mass (with $f \simeq 1$ ). This, of course, is the Higgs mechanism which generates the Wboson mass. The only difference is that here the W-boson is expressed by the $\mathrm{Wu}$-Yang potential and the Higgs coupling becomes magnetic $\left(\vec{C}_{\mu}\right.$ contains the extra factor $\left.1 / g\right)$.

Similar mechanism works for the Weinberg-Salam model. Here again $\vec{A}_{\mu}$ (with $A=B=0$ ) of the ansatz (5) is identical to (28), and we have

$$
\begin{aligned}
D_{\mu} \xi= & i\left(g f \vec{C}_{\mu}+(1-\cos \theta) \partial_{\mu} \phi \hat{r}\right) \cdot \frac{\vec{\tau}}{2} \xi \\
\left|\mathcal{D}_{\mu} \xi\right|^{2}= & \left|D_{\mu} \xi\right|^{2}-\left|\xi^{\dagger} D_{\mu} \xi\right|^{2} \\
& -\left(\xi^{\dagger} D_{\mu} \xi-i \frac{g^{\prime}}{2} B_{\mu}\right)^{2}=\frac{1}{4} g^{2} f^{2}\left(\vec{C}_{\mu}\right)^{2}, \\
\left|\mathcal{D}_{\mu} \phi\right|^{2}= & \frac{1}{2}\left(\partial_{\mu} \rho\right)^{2}+\frac{1}{2} \rho^{2}\left|\mathcal{D}_{\mu} \xi\right|^{2} \\
= & \frac{1}{2}\left(\partial_{\mu} \rho\right)^{2}+\frac{1}{8} g^{2} \rho^{2} f^{2}\left(\vec{C}_{\mu}\right)^{2} .
\end{aligned}
$$

This (with $f \simeq 1$ ) tells that the electroweak monopole acquires mass through the Higgs mechanism which generates mass to the W-boson.

Once this is understood, we can use the dimensional argument to predict the monopole energy. Since the monopole mass term in the Lagrangian contributes to the monopole energy in the classical solution we may expect

$E \simeq C \times \frac{4 \pi}{e^{2}} M_{W}, \quad C \simeq 1$

This implies that the monopole mass should be about $1 / \alpha$ times bigger than the electroweak scale, around $10 \mathrm{TeV}$. But this is an order estimate. Now we have to know how to estimate $C$.

\subsection{Scaling argument}

We can use the Derrick scaling argument to estimate the constant $C$ in (31), assuming the existence of a finite energy monopole solution. If a finite energy monopole does exist, the action principle tells that it should be stable under the rescaling of its field configuration. So consider such a monopole configuration and let

$$
\begin{aligned}
K_{A} & =\int \mathrm{d}^{3} x \frac{1}{4} \vec{F}_{i j}^{2}, \quad K_{B}=\int \mathrm{d}^{3} x \frac{1}{4} B_{i j}^{2} \\
K_{\phi} & =\int \mathrm{d}^{3} x\left|\mathcal{D}_{i} \phi\right|^{2}, \\
V_{\phi} & =\int \mathrm{d}^{3} x \frac{\lambda}{2}\left(|\phi|^{2}-\frac{\mu^{2}}{\lambda}\right)^{2} .
\end{aligned}
$$

With the ansatz (5) we have (with $A=B=0$ )

$$
\begin{aligned}
K_{A} & =\frac{4 \pi}{g^{2}} \int_{0}^{\infty}\left\{\dot{f}^{2}+\frac{\left(f^{2}-1\right)^{2}}{2 r^{2}}\right\} \mathrm{d} r, \\
K_{B} & =\frac{2 \pi}{g^{\prime 2}} \int_{0}^{\infty} \frac{1}{r^{2}} \mathrm{~d} r, \quad K_{\phi}=2 \pi \int_{0}^{\infty}(r \dot{\rho})^{2} \mathrm{~d} r \\
V_{\phi} & =\frac{\pi}{2} \int_{0}^{\infty} \lambda r^{2}\left(\rho^{2}-\rho_{0}^{2}\right)^{2} \mathrm{~d} r .
\end{aligned}
$$

Notice that $K_{B}$ makes the monopole energy infinite. 
Now, consider the spatial scale transformation

$\vec{x} \longrightarrow \lambda \vec{x}$.

Under this we have

$$
\begin{aligned}
& \vec{A}_{k}(\vec{x}) \rightarrow \lambda \vec{A}_{k}(\lambda \vec{x}), \quad B_{k}(\vec{x}) \rightarrow \lambda B_{k}(\lambda \vec{x}), \\
& \phi(\vec{x}) \rightarrow \phi(\lambda \vec{x}),
\end{aligned}
$$

so that

$K_{A} \longrightarrow \lambda K_{A}, \quad K_{B} \longrightarrow \lambda K_{B}$,

$K_{\phi} \longrightarrow \lambda^{-1} K_{\phi}, \quad V_{\phi} \longrightarrow \lambda^{-3} V_{\phi}$.

With this we have the following requirement for the stable monopole configuration:

$K_{A}+K_{B}=K_{\phi}+3 V_{\phi}$.

From this we can estimate the finite value of $K_{B}$.

Now, for the Cho-Maison monopole we have (with $M_{W} \simeq$ $80.4 \mathrm{GeV}, M_{H} \simeq 125 \mathrm{GeV}$, and $\sin ^{2} \theta_{\mathrm{w}}=0.2312$ )

$K_{A} \simeq 0.1904 \times \frac{4 \pi}{e^{2}} M_{W}, \quad K_{\phi} \simeq 0.1577 \times \frac{4 \pi}{e^{2}} M_{W}$,

$V_{\phi} \simeq 0.0111 \times \frac{4 \pi}{e^{2}} M_{W}$.

This, with (37), tells that

$K_{B} \simeq 0.0006 \times \frac{4 \pi}{e^{2}} M_{W}$.

From this we estimate the energy of the monopole to be

$E \simeq 0.3598 \times \frac{4 \pi}{e^{2}} M_{W} \simeq 3.96 \mathrm{TeV}$.

This strongly endorses the dimensional argument. In particular, this tells that the electroweak monopole of mass around a few $\mathrm{TeV}$ could be possible.

The important question now is to show how the quantum correction could actually make the energy of the ChoMaison monopole finite. To do that we have to understand the structure of the electroweak theory, in particular the Abelian decomposition of the electroweak theory. So we review the gauge independent Abelian decomposition of the standard model first.

\section{Abelian decomposition of the electroweak theory}

Consider the Yang-Mills theory

$\mathcal{L}_{Y M}=-\frac{1}{4} \vec{F}_{\mu \nu}^{2}$.

The best way to make the Abelian decomposition is to introduce a unit $S U(2)$ triplet $\hat{n}$ which selects the Abelian direction at each space-time point, and impose the isometry on the gauge potential which determines the restricted potential $\hat{A}_{\mu}$ [36-39]

$D_{\mu} \hat{n}=0$,

$\vec{A}_{\mu} \rightarrow \hat{A}_{\mu}=A_{\mu} \hat{n}-\frac{1}{g} \hat{n} \times \partial_{\mu} \hat{n}=A_{\mu} \hat{n}+\vec{C}_{\mu}$,

$A_{\mu}=\hat{n} \cdot \vec{A}_{\mu}, \quad \vec{C}_{\mu}=-\frac{1}{g} \hat{n} \times \partial_{\mu} \hat{n}$.

Notice that the restricted potential is precisely the connection which leaves $\hat{n}$ invariant under parallel transport. The restricted potential is called a Cho connection or a ChoDuan-Ge (CDG) connection [40-45].

With this we obtain the gauge independent Abelian decomposition of the $S U(2)$ gauge potential adding the valence potential $\vec{W}_{\mu}$ which was excluded by the isometry [36-39]

$\vec{A}_{\mu}=\hat{A}_{\mu}+\vec{W}_{\mu}, \quad\left(\hat{n} \cdot \vec{W}_{\mu}=0\right)$.

The Abelian decomposition has recently been referred to as Cho (also Cho-Duan-Ge or Cho-Faddeev-Niemi) decomposition [40-45].

Under the infinitesimal gauge transformation

$\delta \hat{n}=-\vec{\alpha} \times \hat{n}, \quad \delta \vec{A}_{\mu}=\frac{1}{g} D_{\mu} \vec{\alpha}$,

we have

$\delta A_{\mu}=\frac{1}{g} \hat{n} \cdot \partial_{\mu} \vec{\alpha}, \quad \delta \hat{A}_{\mu}=\frac{1}{g} \hat{D}_{\mu} \vec{\alpha}$,

$\delta \vec{W}_{\mu}=-\vec{\alpha} \times \vec{W}_{\mu}$.

This tells that $\hat{A}_{\mu}$ by itself describes an $S U(2)$ connection which enjoys the full $S U(2)$ gauge degrees of freedom. Furthermore the valence potential $\vec{W}_{\mu}$ forms a gauge covariant vector field under the gauge transformation. But what is really remarkable is that the decomposition is gauge independent. Once $\hat{n}$ is chosen, the decomposition follows automatically, regardless of the choice of gauge.

Notice that $\hat{A}_{\mu}$ has a dual structure,

$$
\begin{aligned}
\hat{F}_{\mu \nu} & =\partial_{\mu} \hat{A}_{\nu}-\partial_{\nu} \hat{A}_{\mu}+g \hat{A}_{\mu} \times \hat{A}_{\nu}=\left(F_{\mu \nu}+H_{\mu \nu}\right) \hat{n}, \\
F_{\mu \nu} & =\partial_{\mu} A_{\nu}-\partial_{\nu} A_{\mu}, \\
H_{\mu \nu} & =-\frac{1}{g} \hat{n} \cdot\left(\partial_{\mu} \hat{n} \times \partial_{\nu} \hat{n}\right) .
\end{aligned}
$$

Moreover, $H_{\mu \nu}$ always admits the potential because it satisfies the Bianchi identity. In fact, replacing $\hat{n}$ with a $C P^{1}$ field $\xi$ (with $\hat{n}=-\xi^{\dagger} \vec{\tau} \xi$ ) we have

$$
\begin{aligned}
& H_{\mu \nu}=\partial_{\mu} \tilde{C}_{\nu}-\partial_{\nu} \tilde{C}_{\mu}=\frac{2 i}{g}\left(\partial_{\mu} \xi^{\dagger} \partial_{\nu} \xi-\partial_{\nu} \xi^{\dagger} \partial_{\mu} \xi\right), \\
& \tilde{C}_{\mu}=\frac{2 i}{g} \xi^{\dagger} \partial_{\mu} \xi=\frac{i}{g}\left(\xi^{\dagger} \partial_{\mu} \xi-\partial_{\mu} \xi^{\dagger} \xi\right) .
\end{aligned}
$$


Of course $\tilde{C}_{\mu}$ is determined uniquely up to the $U(1)$ gauge freedom which leaves $\hat{n}$ invariant. To understand the meaning of $\tilde{C}_{\mu}$, notice that with $\hat{n}=\hat{r}$ we have

$\tilde{C}_{\mu}=\frac{1}{g}(1-\cos \theta) \partial_{\mu} \varphi$.

This is nothing but the Abelian monopole potential, and the corresponding non-Abelian monopole potential is given by the Wu-Yang monopole potential $\vec{C}_{\mu}$ [13-16]. This justifies us to call $A_{\mu}$ and $\tilde{C}_{\mu}$ the electric and magnetic potential.

The above analysis tells that $\hat{A}_{\mu}$ retains all essential topological characteristics of the original non-Abelian potential. First, $\hat{n}$ defines $\pi_{2}\left(S^{2}\right)$ which describes the non-Abelian monopoles. Second, it characterizes the Hopf invariant $\pi_{3}\left(S^{2}\right) \simeq \pi_{3}\left(S^{3}\right)$ which describes the topologically distinct vacua [46-48]. Moreover, it provides the gauge independent separation of the monopole field from the generic non-Abelian gauge potential.

With the decomposition (43), we have

$$
\vec{F}_{\mu \nu}=\hat{F}_{\mu \nu}+\hat{D}_{\mu} \vec{W}_{\nu}-\hat{D}_{\nu} \vec{W}_{\mu}+g \vec{W}_{\mu} \times \vec{W}_{\nu},
$$

so that the Yang-Mills Lagrangian is expressed as

$$
\begin{aligned}
\mathcal{L}_{Y M}= & -\frac{1}{4} \hat{F}_{\mu \nu}^{2}-\frac{1}{4}\left(\hat{D}_{\mu} \vec{W}_{\nu}-\hat{D}_{\nu} \vec{W}_{\mu}\right)^{2} \\
& -\frac{g}{2} \hat{F}_{\mu \nu} \cdot\left(\vec{W}_{\mu} \times \vec{W}_{\nu}\right)-\frac{g^{2}}{4}\left(\vec{W}_{\mu} \times \vec{W}_{\nu}\right)^{2} .
\end{aligned}
$$

This shows that the Yang-Mills theory can be viewed as a restricted gauge theory made of the restricted potential, which has the valence gluons as its source [36-39].

An important advantage of the decomposition (43) is that it can actually Abelianize (or more precisely "dualize") the non-Abelian gauge theory gauge independently [36-39]. To see this let $\left(\hat{n}_{1}, \hat{n}_{2}, \hat{n}\right)$ be a right-handed orthonormal basis of $S U(2)$ space and let

$$
\begin{aligned}
\vec{W}_{\mu}= & W_{\mu}^{1} \hat{n}_{1}+W_{\mu}^{2} \hat{n}_{2}, \\
& \left(W_{\mu}^{1}=\hat{n}_{1} \cdot \vec{W}_{\mu}, \quad W_{\mu}^{2}=\hat{n}_{2} \cdot \vec{W}_{\mu}\right) .
\end{aligned}
$$

With this we have

$$
\begin{aligned}
\hat{D}_{\mu} \vec{W}_{v}= & {\left[\partial_{\mu} W_{\nu}^{1}-g\left(A_{\mu}+\tilde{C}_{\mu}\right) W_{\nu}^{2}\right] \hat{n}_{1} } \\
& +\left[\partial_{\mu} W_{v}^{2}+g\left(A_{\mu}+\tilde{C}_{\mu}\right) W_{\nu}^{1}\right] \hat{n}_{2},
\end{aligned}
$$

so that with

$$
\mathcal{A}_{\mu}=A_{\mu}+\tilde{C}_{\mu}, \quad W_{\mu}=\frac{1}{\sqrt{2}}\left(W_{\mu}^{1}+i W_{\mu}^{2}\right),
$$

we can express the Lagrangian explicitly in terms of the dual potential $\mathcal{A}_{\mu}$ and the complex vector field $W_{\mu}$,

$$
\begin{aligned}
\mathcal{L}_{Y M}= & -\frac{1}{4} \mathcal{F}_{\mu \nu}^{2}-\frac{1}{2}\left|\hat{D}_{\mu} W_{\nu}-\hat{D}_{\nu} W_{\mu}\right|^{2} \\
& +i g \mathcal{F}_{\mu \nu} W_{\mu}^{*} W_{\nu}+\frac{g^{2}}{4}\left(W_{\mu}^{*} W_{\nu}-W_{\nu}^{*} W_{\mu}\right)^{2},
\end{aligned}
$$

where $\mathcal{F}_{\mu \nu}=F_{\mu \nu}+H_{\mu \nu}$ and $\hat{D}_{\mu}=\partial_{\mu}+i g \mathcal{A}_{\mu}$. This shows that we can indeed Abelianize the non-Abelian theory with our decomposition.

Notice that in the Abelian formalism the Abelian potential $\mathcal{A}_{\mu}$ has the extra magnetic potential $\tilde{C}_{\mu}$. In other words, it is given by the sum of the electric and magnetic potentials $A_{\mu}+\tilde{C}_{\mu}$. Clearly $\tilde{C}_{\mu}$ represents the topological degrees of the non-Abelian symmetry which does not show up in the naive Abelianization that one obtains by fixing the gauge [36-39].

Furthermore, this Abelianization is gauge independent, because here we have never fixed the gauge to obtain this Abelian formalism. So one might ask how the non-Abelian gauge symmetry is realized in this Abelian formalism. To discuss this let

$$
\begin{aligned}
\vec{\alpha} & =\alpha_{1} \hat{n}_{1}+\alpha_{2} \hat{n}_{2}+\theta \hat{n}, \quad \alpha=\frac{1}{\sqrt{2}}\left(\alpha_{1}+i \alpha_{2}\right), \\
\vec{C}_{\mu} & =-\frac{1}{g} \hat{n} \times \partial_{\mu} \hat{n}=-C_{\mu}^{1} \hat{n}_{1}-C_{\mu}^{2} \hat{n}_{2}, \\
C_{\mu} & =\frac{1}{\sqrt{2}}\left(C_{\mu}^{1}+i C_{\mu}^{2}\right) .
\end{aligned}
$$

Certainly the Lagrangian (52) is invariant under the active (classical) gauge transformation (45) described by

$\delta A_{\mu}=\frac{1}{g} \partial_{\mu} \theta-i\left(C_{\mu}^{*} \alpha-C_{\mu} \alpha^{*}\right)$,

$\delta \tilde{C}_{\mu}=-\delta A_{\mu}, \quad \delta W_{\mu}=0$.

But it has another gauge invariance, the invariance under the following passive (quantum) gauge transformation:

$\begin{aligned} \delta A_{\mu} & =\frac{1}{g} \partial_{\mu} \theta-i\left(W_{\mu}^{*} \alpha-W_{\mu} \alpha^{*}\right), \\ \delta \tilde{C}_{\mu} & =0, \quad \delta W_{\mu}=\frac{1}{g} \hat{D}_{\mu} \alpha-i \theta W_{\mu} .\end{aligned}$

Clearly this passive gauge transformation assures the desired non-Abelian gauge symmetry for the Abelian formalism. This tells that the Abelian theory not only retains the original gauge symmetry, but actually has an enlarged (both active and passive) gauge symmetries.

The reason for this extra (quantum) gauge symmetry is that the Abelian decomposition automatically puts the theory in the background field formalism which doubles the gauge symmetry [49]. This is because in this decomposition we can view the restricted and valence potentials as the classical and quantum potentials, so that we have freedom to assign the gauge symmetry either to the classical field or to the quantum field. This is why we have the extra gauge symmetry.

The Abelian decomposition has played a crucial role in QCD to demonstrate the Abelian dominance and the monopole condensation in color confinement [50-58]. This is because it separates not only the Abelian potential but also the monopole potential gauge independently. 
Now, consider the Georgi-Glashow model (27). With

$\vec{\Phi}=\rho \hat{n}, \quad \vec{A}_{\mu}=\hat{A}_{\mu}+\vec{W}_{\mu}$,

we have the Abelian decomposition,

$$
\begin{aligned}
\mathcal{L}_{G G}= & -\frac{1}{2}\left(\partial_{\mu} \rho\right)^{2}-\frac{g^{2}}{2} \rho^{2}\left(\vec{W}_{\mu}\right)^{2}-\frac{\lambda}{4}\left(\rho^{2}-\frac{\mu^{2}}{\lambda}\right)^{2} \\
& -\frac{1}{4} \hat{F}_{\mu \nu}^{2}-\frac{1}{4}\left(\hat{D}_{\mu} \vec{W}_{\nu}-\hat{D}_{\nu} \vec{W}_{\mu}\right)^{2} \\
& -\frac{g}{2} \hat{F}_{\mu \nu} \cdot\left(\vec{W}_{\mu} \times \vec{W}_{\nu}\right)-\frac{g^{2}}{4}\left(\vec{W}_{\mu} \times \vec{W}_{\nu}\right)^{2} .
\end{aligned}
$$

With this we can Abelianize it gauge independently,

$$
\begin{aligned}
\mathcal{L}_{G G}= & -\frac{1}{2}\left(\partial_{\mu} \rho\right)^{2}-g^{2} \rho^{2}\left|W_{\mu}\right|^{2}-\frac{\lambda}{4}\left(\rho^{2}-\frac{\mu^{2}}{\lambda}\right)^{2} \\
& -\frac{1}{4} \mathcal{F}_{\mu \nu}^{2}-\frac{1}{2}\left|\hat{D}_{\mu} W_{\nu}-\hat{D}_{\nu} W_{\mu}\right|^{2}+i g \mathcal{F}_{\mu \nu} W_{\mu}^{*} W_{\nu} \\
& +\frac{g^{2}}{4}\left(W_{\mu}^{*} W_{\nu}-W_{\nu}^{*} W_{\mu}\right)^{2} .
\end{aligned}
$$

This clearly shows that the theory can be viewed as a (nontrivial) Abelian gauge theory which has a charged vector field as a source.

The Abelianized Lagrangian looks very much like the Georgi-Glashow Lagrangian written in the unitary gauge. But we emphasize that this is the gauge independent Abelianization which has the full (quantum) $S U(2)$ gauge symmetry.

Obviously we can apply the same Abelian decomposition to the Weinberg-Salam theory

$$
\begin{aligned}
\mathcal{L}= & -\frac{1}{2}\left(\partial_{\mu} \rho\right)^{2}-\frac{\rho^{2}}{2}\left|\hat{\mathcal{D}}_{\mu} \xi\right|^{2}-\frac{\lambda}{8}\left(\rho^{2}-\rho_{0}^{2}\right)^{2} \\
& -\frac{1}{4} \hat{F}_{\mu \nu}^{2}-\frac{1}{4} G_{\mu \nu}^{2}-\frac{1}{4}\left(\hat{D}_{\mu} \vec{W}_{\nu}-\hat{D}_{\nu} \vec{W}_{\mu}\right)^{2} \\
& -\frac{g^{2}}{8} \rho^{2}\left(\vec{W}_{\mu}\right)^{2}-\frac{g}{2} \hat{F}_{\mu \nu} \cdot\left(\vec{W}_{\mu} \times \vec{W}_{\nu}\right) \\
& -\frac{g^{2}}{4}\left(\vec{W}_{\mu} \times \vec{W}_{\nu}\right)^{2} \\
\hat{\mathcal{D}}_{\mu}= & \partial_{\mu}-i \frac{g}{2} \vec{\tau} \cdot \hat{A}_{\mu}-i \frac{g^{\prime}}{2} B_{\mu} .
\end{aligned}
$$

Moreover, with

$$
\left(\begin{array}{c}
A_{\mu}^{(\mathrm{em})} \\
Z_{\mu}
\end{array}\right)=\frac{1}{\sqrt{g^{2}+g^{\prime 2}}}\left(\begin{array}{cc}
g & g^{\prime} \\
-g^{\prime} & g
\end{array}\right)\left(\begin{array}{c}
B_{\mu} \\
\mathcal{A}_{\mu}
\end{array}\right),
$$

we can Abelianize it gauge independently

$$
\begin{aligned}
\mathcal{L}= & -\frac{1}{2}\left(\partial_{\mu} \rho\right)^{2}-\frac{\lambda}{8}\left(\rho^{2}-\rho_{0}^{2}\right)^{2} \\
& -\frac{1}{4} F_{\mu \nu}^{(\mathrm{em})^{2}}-\frac{1}{4} Z_{\mu \nu}^{2}-\frac{g^{2}}{4} \rho^{2}\left|W_{\mu}\right|^{2}-\frac{g^{2}+g^{\prime 2}}{8} \rho^{2} Z_{\mu}^{2} \\
& -\frac{1}{2}\left|\left(D_{\mu}^{(\mathrm{em})} W_{\nu}-D_{\nu}^{(\mathrm{em})} W_{\mu}\right)+i e \frac{g}{g^{\prime}}\left(Z_{\mu} W_{\nu}-Z_{\nu} W_{\mu}\right)\right|^{2}
\end{aligned}
$$

$$
\begin{aligned}
& +i e F_{\mu \nu}^{(\mathrm{em})} W_{\mu}^{*} W_{\nu}+i e \frac{g}{g^{\prime}} Z_{\mu \nu} W_{\mu}^{*} W_{\nu} \\
& +\frac{g^{2}}{4}\left(W_{\mu}^{*} W_{\nu}-W_{\nu}^{*} W_{\mu}\right)^{2},
\end{aligned}
$$

where $D_{\mu}^{(\mathrm{em})}=\partial_{\mu}+i e A_{\mu}^{(\mathrm{em})}$. Again we emphasize that this is not the Weinberg-Salam Lagrangian in the unitary gauge. This is the gauge independent Abelianization which has the extra quantum (passive) non-Abelian gauge degrees of freedom. This can easily be understood comparing (60) with (8). Certainly (60) is gauge independent, while (8) applies to the unitary gauge.

This provides us important piece of information. In the absence of the electromagnetic interaction (i.e., with $A_{\mu}^{(\mathrm{em})}=$ $\left.W_{\mu}=0\right)$ the Weinberg-Salam model describes a spontaneously broken $U(1)_{Z}$ gauge theory,

$\mathcal{L}=-\frac{1}{2}\left(\partial_{\mu} \rho\right)^{2}-\frac{\lambda}{8}\left(\rho^{2}-\rho_{0}^{2}\right)^{2}-\frac{1}{4} Z_{\mu \nu}^{2}-\frac{g^{2}+g^{\prime 2}}{8} \rho^{2} Z_{\mu}^{2}$,

which is nothing but the Ginzburg-Landau theory of superconductivity. Furthermore, here $M_{H}$ and $M_{Z}$ corresponds to the coherence length (of the Higgs field) and the penetration length (of the magnetic field made of $Z$-field). So, when $M_{H}>M_{Z}$ (or $\left.M_{H}<M_{Z}\right)$, the theory describes a type II (or type I) superconductivity, which is well known to admit the Abrikosov-Nielsen-Olesen vortex solution. This confirms the existence of Nambu's string in Weinberg-Salam model. What Nambu showed was that he could make the string finite by attaching the fractionally charged monopole anti-monopole pair to this string [24,25].

\section{Comparison with Julia-Zee dyon}

The Cho-Maison dyon looks very much like the well-known Julia-Zee dyon in the Georgi-Glashow model. Both can be viewed as the $\mathrm{Wu}$-Yang monopole dressed by the weak boson(s). However, there is a crucial difference. The the Julia-Zee dyon is completely regular and has a finite energy, while the Cho-Maison dyon has a point singularity at the center which makes the energy infinite.

So, to regularize the Cho-Maison dyon it is important to understand the difference between the two dyons. To do that notice that, in the absence of the $Z$-boson, (61) reduces to

$$
\begin{aligned}
\mathcal{L}= & -\frac{1}{2}\left(\partial_{\mu} \rho\right)^{2}-\frac{\lambda}{8}\left(\rho^{2}-\rho_{0}^{2}\right)^{2}-\frac{g^{2}}{4} \rho^{2}\left|W_{\mu}\right|^{2} \\
& -\frac{1}{4} F_{\mu \nu}^{(\mathrm{em})^{2}}-\frac{1}{2}\left|D_{\mu}^{(\mathrm{em})} W_{\nu}-D_{\nu}^{(\mathrm{em})} W_{\mu}\right|^{2} \\
& +i e F_{\mu \nu}^{(\mathrm{em})} W_{\mu}^{*} W_{\nu}+\frac{g^{2}}{4}\left(W_{\mu}^{*} W_{\nu}-W_{\nu}^{*} W_{\mu}\right)^{2} .
\end{aligned}
$$


This should be compared with (58), which shows that the two theories have exactly the same type of interaction in the absence of the $Z$-boson, if we identify $\mathcal{F}_{\mu \nu}$ in (58) with $F_{\mu \nu}^{(\mathrm{em})}$ in (63). The only difference is the coupling strengths of the $W$-boson quartic self-interaction and Higgs interaction of $W$-boson (responsible for the Higgs mechanism). This difference, of course, originates from the fact that the WeinbergSalam model has two gauge coupling constants, while the Georgi-Glashow model has only one.

This tells that, in spite of the fact that the Cho-Maison dyon has infinite energy, it is not very different from the JuliaZee dyon. To amplify this point notice that the spherically symmetric ansatz of the Julia-Zee dyon

$$
\begin{aligned}
\vec{\Phi} & =\rho(r) \hat{r}, \quad \hat{A}_{\mu}=\frac{1}{g} A(r) \partial_{\mu} t \hat{r}-\frac{1}{g} \hat{r} \times \partial_{\mu} \hat{r} \\
\vec{W}_{\mu} & =\frac{1}{g} f(r) \hat{r} \times \partial_{\mu} \hat{r},
\end{aligned}
$$

can be written in the Abelian formalism as

$$
\begin{aligned}
\rho & =\rho(r), \quad W_{\mu}=\frac{i}{g} \frac{f(r)}{\sqrt{2}} e^{i \varphi}\left(\partial_{\mu} \theta+i \sin \theta \partial_{\mu} \varphi\right), \\
\mathcal{A}_{\mu} & =\frac{1}{g} A(r) \partial_{\mu} t-\frac{1}{g}(1-\cos \theta) \partial_{\mu} \varphi .
\end{aligned}
$$

In the absence of the $Z$-boson this is identical to the ansat (9).

With the ansatz we have the following equation for the dyon:

$\ddot{\rho}+\frac{2}{r} \dot{\rho}-2 \frac{f^{2}}{r^{2}} \rho=\lambda\left(\rho^{2}-\frac{\mu^{2}}{\lambda}\right) \rho$,

$\ddot{f}-\frac{f^{2}-1}{r^{2}} f=\left(g^{2} \rho^{2}-A^{2}\right) f$,

$\ddot{A}+\frac{2}{r} \dot{A}-2 \frac{f^{2}}{r^{2}} A=0$.

This should be compared to the equation of motion (10) for the Cho-Maison dyon. They are not very different.

With the boundary condition

$$
\begin{aligned}
& \rho(0)=0, \quad f(0)=1, \quad A(0)=0, \\
& \rho(\infty)=\bar{\rho}_{0}=\sqrt{\mu^{2} / \lambda}, \quad f(\infty)=0, \quad A(\infty)=A_{0},
\end{aligned}
$$

one can integrate (66) and obtain the Julia-Zee dyon which has a finite energy. Notice that the boundary condition $A(0)=0$ and $f(0)=1$ is crucial to make the solutions regular at the origin. This confirms that the Julia-Zee dyon is nothing but the Abelian monopole regularized by $\rho$ and $W_{\mu}$, where the charged vector field adds an extra electric charge to the monopole. Again it must be clear from (66) that, for a given magnetic charge, there are always two dyons with opposite electric charges.

Moreover, for the monopole (and anti-monopole) solution with $A=0$, the equation reduces to the follow- ing Bogomol'nyi-Prasad-Sommerfield equation in the limit $\lambda=0$ :

$\dot{\rho} \pm \frac{1}{g r^{2}}\left(f^{2}-1\right)=0, \quad \dot{f} \pm g \rho f=0$.

This has the analytic solution

$\rho=\bar{\rho}_{0} \operatorname{coth}\left(g \bar{\rho}_{0} r\right)-\frac{1}{e r}, \quad f=\frac{g \bar{\rho}_{0} r}{\sinh \left(g \bar{\rho}_{0} r\right)}$,

which describes the Prasad-Sommerfield monopole [20].

Of course, the Cho-Maison dyon has a non-trivial dressing of the $Z$-boson which is absent in the Julia-Zee dyon. But notice that the $Z$-boson plays no role in the Cho-Maison monopole. This confirms that the Cho-Maison monopole and the 't Hooft-Polyakov monopole are not so different, so that the Cho-Maison monopole could be modified to have finite energy.

For the anti-dyon we can have the following ansatz:

$$
\begin{aligned}
\vec{\Phi} & =\rho(r) \hat{r}^{\prime}, \quad \hat{A}_{\mu}^{\prime}=-\frac{1}{g} A(r) \partial_{\mu} t \hat{r}^{\prime}-\frac{1}{g} \hat{r}^{\prime} \times \partial_{\mu} \hat{r}^{\prime} \\
\vec{W}_{\mu}^{\prime} & =\frac{1}{g} f(r) \hat{r}^{\prime} \times \partial_{\mu} \hat{r}^{\prime}, \\
\hat{r}^{\prime} & =(\sin \theta \cos \phi,-\sin \theta \sin \phi, \cos \theta),
\end{aligned}
$$

or equivalently

$$
\begin{aligned}
\rho^{\prime} & =\rho(r), \quad W_{\mu}=\frac{i}{g} \frac{f(r)}{\sqrt{2}} e^{-i \varphi}\left(\partial_{\mu} \theta-i \sin \theta \partial_{\mu} \varphi\right), \\
\mathcal{A}_{\mu}^{\prime} & =-\frac{1}{g} A(r) \partial_{\mu} t+\frac{1}{g}(1-\cos \theta) \partial_{\mu} \varphi .
\end{aligned}
$$

This ansatz looks different from the popular ansatz described by $\vec{\Phi}=-\rho(r) \hat{r}$, but we can easily show that they are gauge equivalent. With this we have exactly the same equation, Eq. (66), for the anti-dyon, which assures that the theory has both dyon and anti-dyon.

\section{Ultraviolet regularization of Cho-Maison dyon}

Since the Cho-Maison dyon is the only dyon in the standard model, it is impossible to regularize it within the model. However, the Weinberg-Salam model is the "bare" theory which should change to the "effective" theory after the quantum correction, and the "real" electroweak dyon must be the solution of such theory. So we may hope that the quantum correction could regularize the Cho-Maison dyon.

The importance of the quantum correction in classical solutions is best understood in QCD. The "bare" QCD Lagrangian has no confinement, so that the classical solutions of the bare QCD can never describe the quarkonium or hadronic bound states. Only the effective theory can.

To see how the quantum modification could make the energy of the Cho-Maison monopole finite, notice that after 
the quantum correction the coupling constants change to the scale dependent running couplings. So, if this quantum correction makes $1 / g^{\prime 2}$ in $E_{0}$ in (26) vanishing in the short distance limit, the Cho-Maison monopole could have finite energy.

To do that consider the following effective Lagrangian which has the non-canonical kinetic term for the $U(1)_{Y}$ gauge field:

$$
\begin{aligned}
\mathcal{L}_{\text {eff }}= & -\left|\mathcal{D}_{\mu} \phi\right|^{2}-\frac{\lambda}{2}\left(\phi^{2}-\frac{\mu^{2}}{\lambda}\right)^{2}-\frac{1}{4} \vec{F}_{\mu \nu}^{2} \\
& -\frac{1}{4} \epsilon\left(|\phi|^{2}\right) G_{\mu \nu}^{2},
\end{aligned}
$$

where $\epsilon\left(|\phi|^{2}\right)$ is a positive dimensionless function of the Higgs doublet which approaches 1 asymptotically. Clearly $\epsilon$ modifies the permittivity of the $U(1)_{Y}$ gauge field, but the effective action still retains the $S U(2) \times U(1)_{Y}$ gauge symmetry. Moreover, when $\epsilon \rightarrow 1$ asymptotically, the effective action reproduces the standard model.

This type of effective theory which has the field dependent permittivity naturally appears in the non-linear electrodynamics and higher-dimensional unified theory, and has been studied intensively in cosmology to explain the latetime accelerated expansion [59-63].

From (72) we have the equations for $\rho$ and $B_{\mu}$

$$
\begin{aligned}
\partial^{2} \rho & =\left|\mathcal{D}_{\mu} \xi\right|^{2} \rho+\frac{\lambda}{2}\left(\rho^{2}-\rho_{0}^{2}\right) \rho+\frac{1}{2} \epsilon^{\prime} \rho G_{\mu \nu}^{2}, \\
\partial_{\mu} G_{\mu \nu} & =i \frac{g^{\prime}}{2 \epsilon} \rho^{2}\left[\xi^{\dagger} \mathcal{D}_{\nu} \xi-\left(\mathcal{D}_{\nu} \xi\right)^{\dagger} \xi\right]-\frac{\partial_{\mu} \epsilon}{\epsilon} G_{\mu \nu},
\end{aligned}
$$

where $\epsilon^{\prime}=\mathrm{d} \epsilon / \mathrm{d} \rho^{2}$. This changes the dyon equation (10) to

$$
\begin{aligned}
& \ddot{\rho}+\frac{2}{r} \dot{\rho}-\frac{f^{2}}{2 r^{2}} \rho=-\frac{1}{4}(A-B)^{2} \rho+\frac{\lambda}{2}\left(\rho^{2}-\rho_{0}^{2}\right) \rho \\
&+\frac{\epsilon^{\prime}}{g^{\prime 2}}\left(\frac{1}{r^{4}}-\dot{B}^{2}\right) \rho, \\
& \ddot{f}-\frac{f^{2}-1}{r^{2}} f=\left(\frac{g^{2}}{4} \rho^{2}-A^{2}\right) f, \\
& \ddot{A}+\frac{2}{r} \dot{A}-\frac{2 f^{2}}{r^{2}} A=\frac{g^{2}}{4} \rho^{2}(A-B), \\
& \ddot{B}+2\left(\frac{1}{r}+\frac{\epsilon^{\prime}}{\epsilon} \rho \dot{\rho}\right) \dot{B}=-\frac{g^{\prime 2}}{4 \epsilon} \rho^{2}(A-B) .
\end{aligned}
$$

This tells that effectively $\epsilon$ changes the $U(1)_{Y}$ gauge coupling $g^{\prime}$ to the "running" coupling $\bar{g}^{\prime}=g^{\prime} / \sqrt{\epsilon}$. This is because with the rescaling of $B_{\mu}$ to $B_{\mu} / g^{\prime}, g^{\prime}$ changes to $g^{\prime} / \sqrt{\epsilon}$. So, by making $\bar{g}^{\prime}$ infinite (requiring $\epsilon$ vanishing) at the origin, we can regularize the Cho-Maison monopole.

From the equations of motion we find that we need the following condition near the origin to make the monopole energy finite:

$\epsilon \simeq\left(\frac{\rho}{\rho_{0}}\right)^{n}, \quad n>4+2 \sqrt{3} \simeq 7.46$.

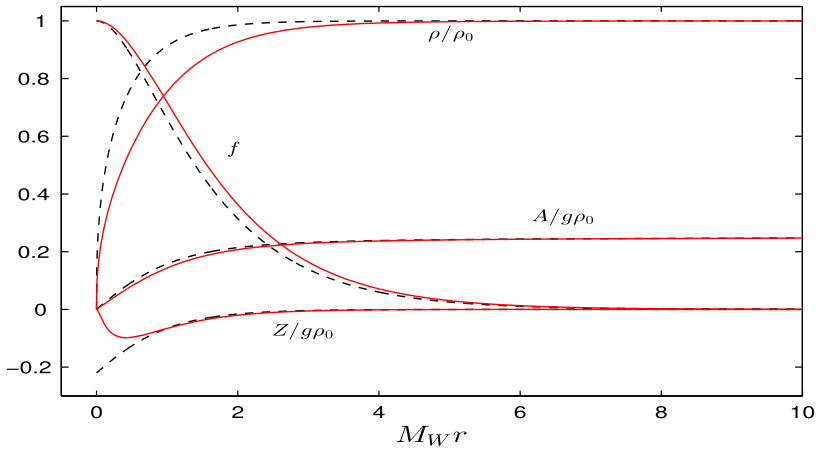

Fig. 1 The finite energy electroweak dyon solution obtained from the effective Lagrangian (72). The solid line represents the finite energy dyon and dotted line represents the Cho-Maison dyon, where $Z=$ $A-B$ and we have chosen $f(0)=1$ and $A(\infty)=M_{W} / 2$

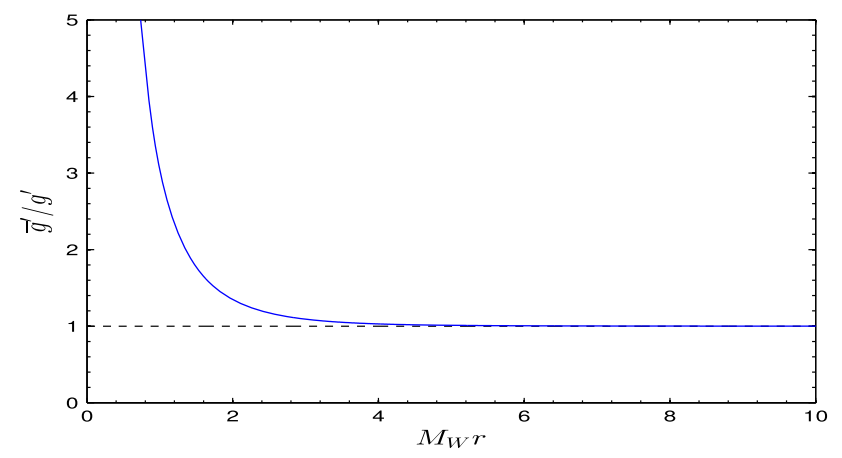

Fig. 2 The running coupling $\bar{g}^{\prime}$ of $U(1)_{Y}$ gauge field induced by the effective Lagrangian (72)

With $n=8$ we have

$\rho(r) \simeq r^{\delta}, \quad \delta=\frac{\sqrt{3}-1}{2}$,

near the origin, and we have the finite energy dyon solution shown in Fig. 1. It is really remarkable that the regularized solutions look very much like the Cho-Maison solutions, except that for the finite energy dyon solution $Z(0)$ becomes 0 . This confirms that the ultraviolet regularization of the ChoMaison monopole is indeed possible.

As expected with $n=8$ the running coupling $\bar{g}^{\prime}$ becomes divergent at the origin, which makes the energy contribution from the $U(1)_{Y}$ gauge field finite. The scale dependence of the running coupling is shown in Fig. 2. With $A=B=0$ we can estimate the monopole energy to be

$E \simeq 0.65 \times \frac{4 \pi}{e^{2}} M_{W} \simeq 7.19 \mathrm{TeV}$.

This tells that the estimate of the monopole energy based on the scaling argument is reliable. The finite energy monopole solution is shown in Fig. 3.

There is another way to regularize the Cho-Maison monopole. Suppose we have the following ultraviolet modi- 


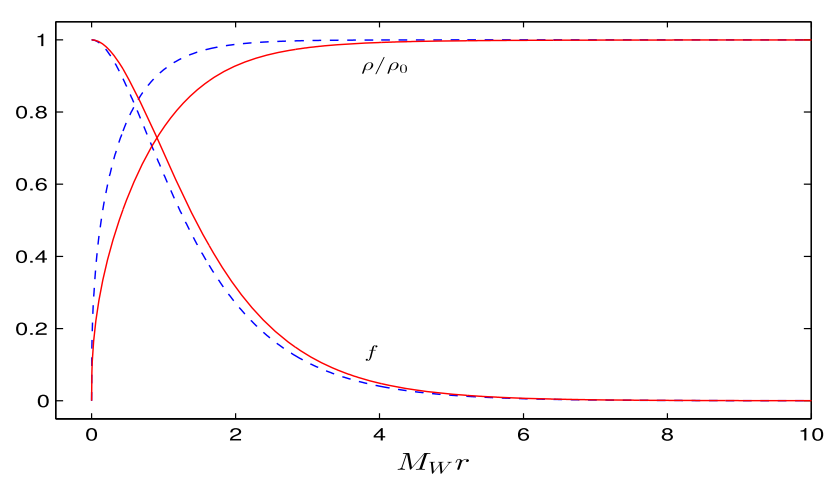

Fig. 3 The finite energy electroweak monopole solution obtained from the effective Lagrangian (79). The solid line (red) represents the regularized monopole and the dotted (blue) line represents the Cho-Maison monopole

fication of (59) from the quantum correction:

$$
\begin{aligned}
\delta \mathcal{L}= & i e \alpha F_{\mu \nu}^{(\mathrm{em})} W_{\mu}^{*} W_{\nu}+\beta \frac{g^{2}}{4}\left(W_{\mu}^{*} W_{\nu}-W_{\nu}^{*} W_{\mu}\right)^{2} \\
& -\gamma \frac{g^{2}}{4} \rho^{2}\left|W_{\mu}\right|^{2},
\end{aligned}
$$

where $\alpha, \beta, \gamma$ are the scale dependent parameters which vanish asymptotically (and modify the theory only at short distance). The justification of these counterterms is clear. The existence of the monopole could affect the $W$-boson magnetic moment and induce the $\alpha$-term. The $\beta$ and $\gamma$ terms could come from the coupling constant and mass renormalizations of the $W$-boson.

Thus we have the modified Weinberg-Salam Lagrangian

$$
\begin{aligned}
\mathcal{L}^{\prime}= & -\frac{1}{2}\left(\partial_{\mu} \rho\right)^{2}-\frac{\lambda}{8}\left(\rho^{2}-\rho_{0}^{2}\right)^{2}-\frac{1}{4} F_{\mu \nu}^{(\mathrm{em})^{2}}-\frac{1}{4} Z_{\mu \nu}^{2} \\
& -\frac{1}{2}\left|\left(D_{\mu}^{(\mathrm{em})} W_{\nu}-D_{\nu}^{(\mathrm{em})} W_{\mu}\right)+i e \frac{g}{g^{\prime}}\left(Z_{\mu} W_{\nu}-Z_{\nu} W_{\mu}\right)\right|^{2} \\
& +i e(1+\alpha) F_{\mu \nu}^{(\mathrm{em})} W_{\mu}^{*} W_{\nu}+i e \frac{g}{g^{\prime}} Z_{\mu \nu} W_{\mu}^{*} W_{\nu} \\
& +(1+\beta) \frac{g^{2}}{4}\left(W_{\mu}^{*} W_{\nu}-W_{\nu}^{*} W_{\mu}\right)^{2} \\
& -(1+\gamma) \frac{g^{2}}{4} \rho^{2}\left|W_{\mu}\right|^{2}-\frac{g^{2}+g^{\prime 2}}{8} \rho^{2} Z_{\mu}^{2} .
\end{aligned}
$$

Of course, this modification is supposed to hold only in the short distance, so that asymptotically $\alpha, \beta, \gamma$ should vanish to make sure that $\mathcal{L}^{\prime}$ reduces to the standard model. But we will treat them as constants, partly because it is difficult to make them scale dependent, but mainly because asymptotically the boundary condition automatically makes them irrelevant and assures the solution to converge to the ChoMaison solution.

To understand the physical meaning of (79) notice that in the absence of the $Z$-boson the above Lagrangian reduces to the Georgi-Glashow Lagrangian where the $W$-boson has an extra "anomalous" magnetic moment $\alpha$ when $(1+\beta)=$ $e^{2} / g^{2}$ and $(1+\gamma)=4 e^{2} / g^{2}$, if we identify the coupling constant $g$ in the Georgi-Glashow model with the electromagnetic coupling constant $e$. Moreover, the ansatz (5) can be written as

$$
\begin{aligned}
\vec{A}_{\mu} & =\hat{A}_{\mu}^{(\mathrm{em})}+\vec{W}_{\mu}, \\
\hat{A}_{\mu}^{(\mathrm{em})} & =e\left[\frac{1}{g^{2}} A(r)+\frac{1}{g^{\prime 2}} B(r)\right] \partial_{\mu} t \hat{r}-\frac{1}{e} \hat{r} \times \partial_{\mu} \hat{r}, \\
\vec{W}_{\mu} & =\frac{f(r)}{g} \hat{r} \times \partial_{\mu} \hat{r}, \\
Z_{\mu} & =\frac{e}{g g^{\prime}}(A(r)-B(r)) \partial_{\mu} t .
\end{aligned}
$$

This shows that, for the monopole (i.e., for $A=B=0$ ), the ansatz becomes formally identical to (64) if $\vec{W}_{\mu}$ is rescaled by a factor $g / e$. This tells that, as far as the monopole solution is concerned, in the absence of the Z-boson the WeinbergSalam model and Georgi-Glashow model are not so different.

With (79) the energy of the dyon is given by

$$
\begin{aligned}
\hat{E}= & \hat{E}_{0}+\hat{E}_{1}, \\
\hat{E}_{0}= & \frac{2 \pi}{g^{2}} \int_{0}^{\infty} \frac{\mathrm{d} r}{r^{2}}\left\{\frac{g^{2}}{g^{\prime 2}}+1-2(1+\alpha) f^{2}+(1+\beta) f^{4}\right\} \\
= & \frac{2 \pi}{g^{2}} \int_{0}^{\infty} \frac{\mathrm{d} r}{r^{2}}\left\{\frac{g^{2}}{e^{2}}-\frac{(1+\alpha)^{2}}{1+\beta}+(1+\beta)\left(f^{2}-\frac{1+\alpha}{1+\beta}\right)^{2}\right\}, \\
\hat{E}_{1}= & \frac{4 \pi}{g^{2}} \int_{0}^{\infty} \mathrm{d} r\left\{\frac{g^{2}}{2}(r \dot{\rho})^{2}+\frac{\lambda g^{2} r^{2}}{8}\left(\rho^{2}-\rho_{0}^{2}\right)^{2}\right. \\
& +\dot{f}^{2}+\frac{1}{2}(r \dot{A})^{2}+\frac{g^{2}}{2 g^{\prime 2}}(r \dot{B})^{2}+(1+\gamma) \frac{g^{2}}{4} f^{2} \rho^{2} \\
& \left.+\frac{g^{2} r^{2}}{8}(B-A)^{2} \rho^{2}+f^{2} A^{2}\right\} .
\end{aligned}
$$

Notice that $\hat{E}_{1}$ remains finite with the modification, and $\gamma$ plays no role to make the monopole energy finite.

To make $\hat{E}_{0}$ finite we must have

$$
1+\alpha=\frac{1}{f(0)^{2}} \frac{g^{2}}{e^{2}}, \quad 1+\beta=\frac{1}{f(0)^{4}} \frac{g^{2}}{e^{2}},
$$

so that the constants $\alpha$ and $\beta$ are fixed by $f(0)$. With this the equation of motion is given by

$$
\begin{aligned}
\ddot{\rho}+\frac{2}{r} \dot{\rho}-\frac{(1+\gamma) f^{2}}{2 r^{2}} \rho & =-\frac{1}{4}(A-B)^{2} \rho+\frac{\lambda}{2}\left(\rho^{2}-\rho_{0}^{2}\right) \rho, \\
\ddot{f}-\frac{(1+\alpha)}{r^{2}}\left(\frac{f^{2}}{f^{2}(0)}-1\right) f & =\left((1+\gamma) \frac{g^{2}}{4} \rho^{2}-A^{2}\right) f, \\
\ddot{A}+\frac{2}{r} \dot{A}-\frac{2 f^{2}}{r^{2}} A & =\frac{g^{2}}{4}(A-B) \rho^{2}, \\
\ddot{B}+\frac{2}{r} \dot{B} & =-\frac{g^{\prime 2}}{4}(A-B) \rho^{2} .
\end{aligned}
$$




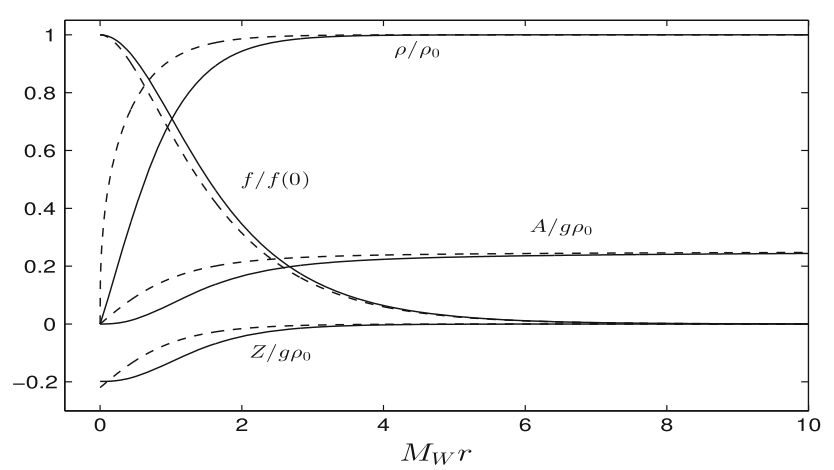

Fig. 4 The finite energy electroweak dyon solution obtained from the modified Lagrangian (79). The solid line represents the finite energy dyon and dotted line represents the Cho-Maison dyon

The solution has the following behavior near the origin:

$\rho \simeq \alpha_{1} r^{\delta_{1}}, \quad \frac{f}{f(0)} \simeq 1+\beta_{1} r^{\delta_{2}}$,
$A \simeq a_{1} r^{\delta_{3}}, \quad B \simeq b_{0}+b_{1} r^{\delta_{4}}$,

where

$\delta_{1}=\frac{1}{2}\left(\sqrt{1+2(1+\gamma) f^{2}(0)}-1\right)$,

$\delta_{2}=\frac{1}{2}(1+\sqrt{8 \alpha+9}), \quad \delta_{3}=\frac{1}{2}\left(\sqrt{1+8 f^{2}(0)}-1\right)$,

$\delta_{4}=\sqrt{1+2 f^{2}(0)}+1$.

Notice that all four deltas are positive (as far as $(1+\alpha)>0)$, so that the four functions are well behaved at the origin.

If we assume $\alpha=\gamma=0$ we have $f(0)=g / e$, and we can integrate (83) with the boundary condition

$\rho(0)=0, \quad f(0)=g / e, \quad A(0)=0, \quad B(0)=b_{0}$,

$f(\infty)=0, \quad \rho(\infty)=\rho_{0}, \quad A(\infty)=B(\infty)=A_{0}$.

The finite energy dyon solution is shown in Fig. 4. It should be emphasized that the solution is an approximate solution which is supposed to be valid only near the origin, because the constants $\alpha, \beta, \gamma$ are supposed to vanish asymptotically. But notice that asymptotically the solution automatically approaches the Cho-Maison solution even without making them vanish, because we have the same boundary condition at the infinity. Again it is remarkable that the finite energy solution looks very similar to the Cho-Maison solution.

Of course, we can still integrate (83) with arbitrary $f(0)$ and have a finite energy solution. The monopole energy for $f(0)=1$ and $f(0)=g / e($ with $\alpha=\gamma=0)$ are given by

$$
\begin{aligned}
& E(f(0)=1) \simeq 0.61 \times \frac{4 \pi}{e^{2}} M_{W} \simeq 6.73 \mathrm{TeV} \\
& E\left(f(0)=\frac{g}{e}\right) \simeq 1.27 \times \frac{4 \pi}{e^{2}} M_{W} \simeq 13.95 \mathrm{TeV}
\end{aligned}
$$

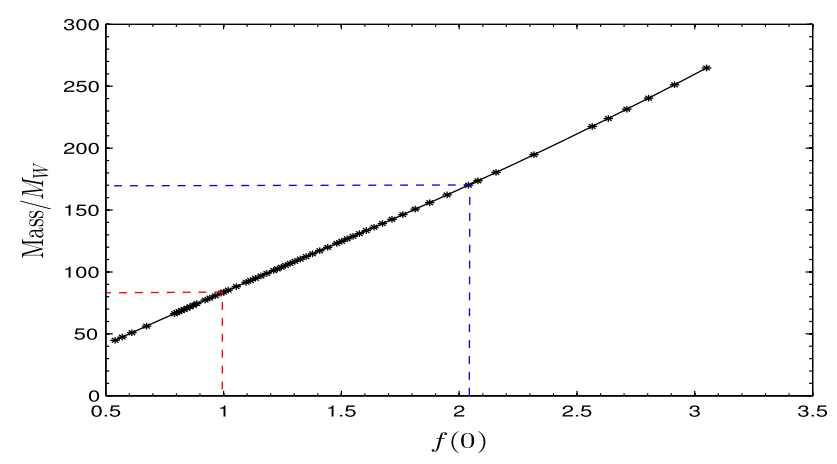

Fig. 5 The energy dependence of the electroweak monopole on $f(0)$

In general the energy of a dyon depends on $f(0)$, but must be of the order of $\left(4 \pi / e^{2}\right) M_{W}$. The energy dependence of the monopole on $f(0)$ is shown in Fig. 5. This strongly supports our prediction of the monopole mass based on the scaling argument.

As we have emphasized, in the absence of the Z-boson (79) reduces to the Georgi-Glashow theory with

$\alpha=0, \quad 1+\beta=\frac{e^{2}}{g^{2}}, \quad 1+\gamma=\frac{4 e^{2}}{g^{2}}$.

In this case (83) reduces to the following Bogomol'nyiPrasad-Sommerfield equation in the limit $\lambda=0$ [20]:

$\dot{\rho} \pm \frac{1}{e r^{2}}\left(\frac{e^{2}}{g^{2}} f^{2}-1\right)=0, \quad \dot{f} \pm e \rho f=0$.

This has the analytic monopole solution

$\rho=\rho_{0} \operatorname{coth}\left(e \rho_{0} r\right)-\frac{1}{e r}, \quad f=\frac{g \rho_{0} r}{\sinh \left(e \rho_{0} r\right)}$,

whose energy is given by the Bogomol'nyi bound

$E=\sin \theta_{\mathrm{w}} \times \frac{8 \pi}{e^{2}} M_{W} \simeq 5.08 \mathrm{TeV}$

From this we can confidently say that the mass of the electroweak monopole could be around 4 to $10 \mathrm{TeV}$.

This confirms that we can regularize the Cho-Maison dyon with a simple modification of the coupling strengths of the existing interactions, which could be caused by the quantum correction. This provides a most economic way to make the energy of the dyon finite without introducing a new interaction in the standard model.

\section{Embedding $U(1)_{Y}$ to $S U(2)_{Y}$}

Another way to regularize the Cho-Maison dyon, of course, is to enlarge $U(1)_{Y}$ and embed it to another $\mathrm{SU}(2)$. This type of generalization of the standard model could naturally arise in the left-right symmetric grand unification models, 
in particular in the $\mathrm{SO}(10)$ grand unification, although this generalization may be too simple to be realistic.

To construct the desired solutions we introduce a hypercharged vector field $X_{\mu}$ and a Higgs field $\sigma$, and we generalize the Lagrangian (59) adding the following Lagrangian:

$$
\begin{aligned}
\Delta \mathcal{L}= & -\frac{1}{2}\left|\tilde{D}_{\mu} X_{v}-\tilde{D}_{\nu} X_{\mu}\right|^{2}+i g^{\prime} G_{\mu \nu} X_{\mu}^{*} X_{v} \\
& +\frac{1}{4} g^{\prime 2}\left(X_{\mu}^{*} X_{v}-X_{\nu}^{*} X_{\mu}\right)^{2} \\
& -\frac{1}{2}\left(\partial_{\mu} \sigma\right)^{2}-g^{\prime 2} \sigma^{2}\left|X_{\mu}\right|^{2}-\frac{\kappa}{4}\left(\sigma^{2}-\frac{m^{2}}{\kappa}\right)^{2},
\end{aligned}
$$

where $\tilde{D}_{\mu}=\partial_{\mu}+i g^{\prime} B_{\mu}$. To understand the meaning of it let us introduce a hypercharge $S U$ (2) gauge field $\vec{B}_{\mu}$ and a scalar triplet $\vec{\Phi}$, and consider the $S U(2)_{Y}$ Georgi-Glashow model

$\mathcal{L}^{\prime}=-\frac{1}{2}\left(D_{\mu} \vec{\Phi}\right)^{2}-\frac{\kappa}{4}\left(\vec{\Phi}^{2}-\frac{m^{2}}{\kappa}\right)^{2}-\frac{1}{4} \vec{G}_{\mu \nu}^{2}$.

Now we can have the Abelian decomposition of this Lagrangian, with $\vec{\Phi}=\sigma \hat{n}$, and have (identifying $B_{\mu}$ and $X_{\mu}$ as the Abelian and valence parts)

$\mathcal{L}^{\prime}=-\frac{1}{4} G_{\mu \nu}^{2}+\Delta \mathcal{L}$.

This clearly shows that Lagrangian (91) describes nothing but the embedding of the hypercharge $U(1)$ to an $S U(2)$ Georgi-Glashow model.

Now for a static spherically symmetric ansatz we choose (5) and let

$$
\begin{aligned}
\sigma & =\sigma(r), \\
X_{\mu} & =\frac{i}{g^{\prime}} \frac{h(r)}{\sqrt{2}} e^{i \varphi}\left(\partial_{\mu} \theta+i \sin \theta \partial_{\mu} \varphi\right) .
\end{aligned}
$$

With the spherically symmetric ansatz the equations of motion are reduced to

$$
\begin{aligned}
\ddot{f}-\frac{f^{2}-1}{r^{2}} f & =\left(\frac{g^{2}}{4} \rho^{2}-A^{2}\right) f, \\
\ddot{\rho}+\frac{2}{r} \dot{\rho}-\frac{f^{2}}{2 r^{2}} \rho & =-\frac{1}{4}(A-B)^{2} \rho+\frac{\lambda}{2}\left(\rho^{2}-\frac{2 \mu^{2}}{\lambda}\right) \rho, \\
\ddot{A}+\frac{2}{r} \dot{A}-\frac{2 f^{2}}{r^{2}} A & =\frac{g^{2}}{4} \rho^{2}(A-B), \\
\ddot{B}+\frac{2}{r} \dot{B}-\frac{2 h^{2}}{r^{2}} B & =\frac{g^{\prime 2}}{4} \rho^{2}(B-A), \\
\ddot{h}-\frac{h^{2}-1}{r^{2}} h & =\left(g^{\prime 2} \sigma^{2}-B^{2}\right) h, \\
\ddot{\sigma}+\frac{2}{r} \dot{\sigma}-\frac{2 h^{2}}{r^{2}} \sigma & =\kappa\left(\sigma^{2}-\frac{m^{2}}{\kappa}\right) \sigma .
\end{aligned}
$$

Furthermore, the energy of the above configuration is given by

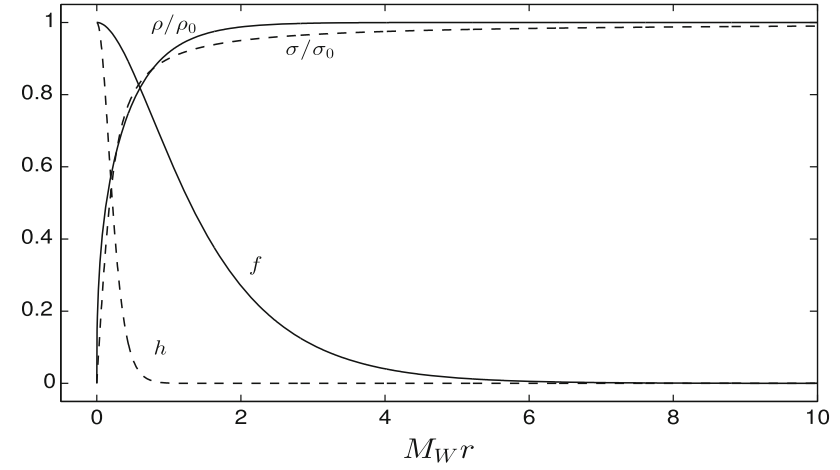

Fig. 6 The $S U(2) \times S U(2)$ monopole solution with $M_{H} / M_{W}=1.56$, $M_{X}=10 M_{W}$, and $\kappa=0$

$$
\begin{aligned}
E= & E_{W}+E_{X}, \\
E_{W}= & \frac{4 \pi}{g^{2}} \int_{0}^{\infty} \mathrm{d} r\left\{\dot{f}^{2}+\frac{\left(f^{2}-1\right)^{2}}{2 r^{2}}+\frac{1}{2}(r \dot{A})^{2}\right. \\
& +f^{2} A^{2}+\frac{g^{2}}{2}(r \dot{\rho})^{2}+\frac{g^{2}}{4} f^{2} \rho^{2}+\frac{g^{2} r^{2}}{8}(A-B)^{2} \rho^{2} \\
& \left.+\frac{\lambda g^{2} r^{2}}{8}\left(\rho^{2}-\frac{2 \mu^{2}}{\lambda}\right)^{2}\right\}=\frac{4 \pi}{g^{2}} C_{1} M_{W}, \\
E_{X}= & \frac{4 \pi}{g^{\prime 2}} \int_{0}^{\infty} \mathrm{d} r\left\{\dot{h}^{2}+\frac{\left(h^{2}-1\right)^{2}}{2 r^{2}}+\frac{1}{2}(r \dot{B})^{2}\right. \\
& +h^{2} B^{2}+\frac{g^{\prime 2}}{2}(r \dot{\sigma})^{2}+g^{\prime 2} h^{2} \sigma^{2} \\
& \left.+\frac{\kappa g^{\prime 2} r^{2}}{4}\left(\sigma^{2}-\sigma_{0}^{2}\right)^{2}\right\}=\frac{4 \pi}{g^{\prime 2}} C_{2} M_{X},
\end{aligned}
$$

where $\sigma_{0}=\sqrt{m^{2} / \kappa}, M_{X}=g^{\prime} \sigma_{0}$, and $C_{1}$ and $C_{2}$ are constants of the order 1 . The boundary conditions for a regular field configuration can be chosen as

$$
\begin{aligned}
& f(0)=h(0)=1, \quad A(0)=B(0)=\rho(0)=\sigma(0)=0, \\
& f(\infty)=h(\infty)=0, \quad A(\infty)=A_{0}, B(\infty)=B_{0}, \\
& \rho(\infty)=\rho_{0}, \quad \sigma(\infty)=\sigma_{0} .
\end{aligned}
$$

Notice that this guarantees the analyticity of the solution everywhere, including the origin.

With the boundary condition (97) one may try to find the desired solution. From the physical point of view one could assume $M_{X} \gg M_{W}$, where $M_{X}$ is an intermediate scale which lies somewhere between the grand unification scale and the electroweak scale. Now, let $A=B=0$ for simplicity. Then (95) decouples to describe two independent systems so that the monopole solution has two cores, the one with the size $O\left(1 / M_{W}\right)$ and the other with the size $O\left(1 / M_{X}\right)$. With $M_{X}=10 M_{W}$ we obtain the solution shown in Fig. 6 in the limit $\kappa=0$ and $M_{H} / M_{W}=1.56$.

In this limit we find $C_{1}=1.53$ and $C_{2}=1$, so that the energy of the solution is given by 


$$
\begin{aligned}
E & =\frac{4 \pi}{e^{2}}\left(\cos ^{2} \theta_{\mathrm{w}}+0.153 \sin ^{2} \theta_{\mathrm{w}}\right) M_{X} \\
& \simeq 110.17 M_{X} .
\end{aligned}
$$

Clearly the solution describes the Cho-Maison monopole whose singularity is regularized by a Prasad-Sommerfield monopole of the size $O\left(1 / M_{X}\right)$.

Notice that, even though the energy of the monopole is fixed by the intermediate scale, the size of the monopole is determined by the electroweak scale. Furthermore from the outside the monopole looks exactly the same as the ChoMaison monopole. Only the inner core is regularized by the hypercharged vector field.

\section{Conclusions}

In this paper we have discussed three ways to estimate the mass of the electroweak monopole, the dimensional argument, the scaling argument, and the ultraviolet regularization of the Cho-Maison monopole. As importantly, we have shown that the standard model has the anti-dyon as well as the dyon solution, so that they can be produced in pairs.

It has generally been believed that the finite energy monopole could exist only at the grand unification scale [21]. But our result tells that the genuine electroweak monopole of mass around 4 to $10 \mathrm{TeV}$ could exist. This strongly implies that there is an excellent chance that MoEDAL could actually detect such monopole in the near future, because the $14 \mathrm{TeV}$ LHC upgrade now reaches the monopole-anti-monopole pair production threshold. But of course, if the mass of the monopole exceeds the LHC threshold $7 \mathrm{TeV}$, we may have to look for the monopole from cosmic ray with the "cosmic" MoEDAL.

The importance of the electroweak monopole is that it is the electroweak generalization of the Dirac monopole, and that it is the only realistic monopole which can be produced and detected. A remarkable aspect of this monopole is that mathematically it can be viewed as a hybrid between the Dirac monopole and the 't Hooft-Polyakov monopole.

However, there are two crucial differences. First, the magnetic charge of the electroweak monopole is two times bigger than that of the Dirac monopole, so that it satisfies the Schwinger quantization condition $q_{m}=4 \pi n / e$. This is because the electroweak generalization requires us to embed $U(1)_{\mathrm{em}}$ to the $\mathrm{U}(1)$ subgroup of $\mathrm{SU}(2)$, which has the period of $4 \pi$. So the magnetic charge of the electroweak monopole has the unit $4 \pi / e$.

Of course, the finite energy dyon solutions we discussed above are not the solutions of the "bare" standard model. Nevertheless they tell us how the Cho-Maison dyon could be regularized and how the regularized electroweak dyon would look like. From the physical point of view there is no doubt that the finite energy solutions should be interpreted as the regularized Cho-Maison dyons whose mass and size are fixed by the electroweak scale.

We emphasize that, unlike Dirac's monopole, which can exist only when $U(1)_{\mathrm{em}}$ becomes non-trivial, the electroweak monopole must exist in the standard model. So, if the standard model is correct, we must have the monopole. In this sense, the experimental discovery of the electroweak monopole should be viewed as the final topological test of the standard model.

At this point it is worth mentioning other closely related topological solutions of the standard model, in particular the sphaleron which could induce the baryon number violation [29-31,64,65]. As we have already remarked the sphaleron and our monopole share the same topology. In this sense the discovery of the sphaleron could provide another topological test of the standard model. On the other hand the sphaleron has an intrinsic instability because it is a saddle point solution, while our monopole has the topological stability. This makes it easier to find the monopole.

But they have similar feature. The energy of the sphaleron is estimated to be about the same as the monopole mass, $E_{s} \simeq 3.9 \times 4 \pi / g^{2} M_{W} \simeq 7.25 \mathrm{TeV}[64,65]$. This, of course is not an accident. Clearly our dimensional argument on the monopole mass equally applies to the sphaleron, so that they must have similar energy.

Another related topological object is the electroweak skyrmion [66-68]. But this soliton becomes possible only in the modified standard model which has an extra term motivated by the renormalization, so that it becomes less relevant.

Clearly the electroweak monopole invites more difficult questions. How can we justify the perturbative expansion and the renormalization in the presence of the monopole? What are the new physical processes which can be induced by the monopole? Most importantly, how can we construct the quantum field theory of the monopole?

Moreover, the existence of the finite energy electroweak monopole should have important physical implications. In particular, it could have important implications in cosmology, because it can be produced after the inflation. The physical implications of the monopole will be discussed in a separate paper [69].

Acknowledgments The work is supported in part by the National Research Foundation (2012-002-134) of the Ministry of Science and Technology and by Konkuk University.

Open Access This article is distributed under the terms of the Creative Commons Attribution License which permits any use, distribution, and reproduction in any medium, provided the original author(s) and the source are credited.

Funded by $\mathrm{SCOAP}^{3}$ / License Version CC BY 4.0. 


\section{References}

1. G. Aad et al. (ATLAS Collaboration), Phys. Lett. B 716, 1 (2012)

2. S. Chatrchyan et al. (CMS Collaboration), Phys. Lett. B 716, 30 (2012)

3. T. Aaltonen et al. (CDF and D0 Collaborations), Phys. Rev. Lett. 109, 071804 (2012)

4. Y.M. Cho, D. Maison, Phys. Lett. B 391, 360 (1997)

5. W.S. Bae, Y.M. Cho, JKPS 46, 791 (2005)

6. Y. Yang, Proc. R. Soc. A 454, 155 (1998)

7. Y. Yang, Solitons in Field Theory and Nonlinear Analysis. Springer Monographs in Mathematics (Springer, New York, 2001), p. 322

8. J. Pinfold, Radiat. Meas. 44, 834 (2009)

9. J. Pinfold, in Progress in High-Energy Physics and Nuclear Safety, ed. by V. Begun et al. (Springer Science and Business Media, Berlin, 2009), p. 217

10. Y.M. Cho, J. Pinfold, Snowmass whitepaper, arXiv:1307.8390 [hep-ph]

11. B. Acharya et al. (MoEDAL Collaboration), Int. J. Mod. Phys. A 29, 1430050 (2014)

12. P.A.M. Dirac, Phys. Rev. 74, 817 (1948)

13. T.T. Wu, C.N. Yang, in Properties of Matter under Unusual Conditions, ed. by H. Mark, S. Fernbach (Interscience, New York, 1969)

14. T.T. Wu, C.N. Yang, Phys. Rev. D 12, 3845 (1975)

15. Y.M. Cho, Phys. Rev. Lett. 44, 1115 (1980)

16. Y.M. Cho, Phys. Lett. B 115, 125 (1982)

17. G. 't Hooft, Nucl. Phys. B 79, 276 (1974)

18. A. Polyakov, JETP Lett. 20, 194 (1974)

19. B. Julia, A. Zee, Phys. Rev. D 11, 2227 (1975)

20. M. Prasad, C. Sommerfield, Phys. Rev. Lett. 35, 760 (1975)

21. C. Dokos, T. Tomaras, Phys. Rev. D 21, 2940 (1980)

22. T. Vachaspati, M. Barriola, Phys. Rev. Lett. 69, 1867 (1992)

23. M. Barriola, T. Vachaspati, M. Bucher, Phys. Rev. D 50, 2819 (1994)

24. Y. Nambu, Nucl. Phys. B 130, 505 (1977)

25. T. Vachaspati, Phys. Rev. Lett. 68, 1977 (1992)

26. T. Vachaspati, Nucl. Phys. B 439, 79 (1995)

27. B. Cabrera, Phys. Rev. Lett. 48, 1378 (1982)

28. P. Forgács, N. Manton, Commun. Math. Phys. 72, 15 (1980)

29. R. Dashen, B. Hasslacher, A. Neveu, Phys. Rev. D 10, 4138 (1974)

30. N. Manton, Phys. Rev. D 28, 2019 (1983)

31. F. Klinkhammer, N. Manton, Phys. Rev. D 30, 2212 (1984)

32. F. Bais, R. Russell, Phys. Rev. D 11, 2692 (1975)

33. Y.M. Cho, P.G.O. Freund, Phys. Rev. D 12, 1711 (1975)
34. Y.M. Cho, D.H. Park, J. Math. Phys. 31, 695 (1990)

35. P. Breitenlohner, P. Forgács, D. Maison, Nucl. Phys. B 383, 357 (1992)

36. Y.M. Cho, Phys. Rev. D 21, 1080 (1980)

37. Y.M. Cho, Phys. Rev. Lett. 46, 302 (1981)

38. Y.M. Cho, Phys. Rev. D 23, 2415 (1981)

39. W.S. Bae, Y.M. Cho, S.W. Kimm, Phys. Rev. D 65, 025005 (2002)

40. L. Faddeev, A. Niemi, Phys. Rev. Lett. 82, 1624 (1999)

41. L. Faddeev, A. Niemi, Phys. Lett. B 449, 214 (1999)

42. S. Shabanov, Phys. Lett. B 458, 322 (1999)

43. S. Shabanov, Phys. Lett. B 463, 263 (1999)

44. H. Gies, Phys. Rev. D 63, 125023 (2001)

45. R. Zucchini, Int. J. Geom. Methods Mod. Phys. 1, 813 (2004)

46. A. Belavin, A. Polyakov, A. Schwartz, Y. Tyupkin, Phys. Lett. B 59, 85 (1975)

47. G. 't Hooft, Phys. Rev. Lett. 37, 8 (1976)

48. Y.M. Cho, Phys. Lett. B 81, 25 (1979)

49. B. DeWitt, Phys. Rev. 162, 1195 (1967)

50. Y.M. Cho, Phys. Rev. D 62, 074009 (2000)

51. Y.M. Cho, D.G. Pak, Phys. Rev. D 65, 074027 (2002)

52. Y.M. Cho, M.L. Walker, D.G. Pak, JHEP 05, 073 (2004)

53. Y.M. Cho, M.L. Walker, Mod. Phys. Lett. A 19, 2707 (2004)

54. Y.M. Cho, F.H. Cho, J.H. Yoon, Phys. Rev. D 87, 085025 (2013)

55. S. Kato, K. Kondo, T. Murakami, A. Shibata, T. Shinohara, S. Ito, Phys. Lett. B 632, 326 (2006)

56. S. Ito, S. Kato, K. Kondo, T. Murakami, A. Shibata, T. Shinohara, Phys. Lett. B 645, 67 (2007)

57. S. Ito, S. Kato, K. Kondo, T. Murakami, A. Shibata, T. Shinohara, Phys. Lett. B 653, 101 (2007)

58. S. Ito, S. Kato, K. Kondo, T. Murakami, A. Shibata, T. Shinohara, Phys. Lett. B 669, 107 (2008)

59. Y.M. Cho, Phys. Rev. D 35, R2628 (1987)

60. Y.M. Cho, Phys. Lett. B 199, 358 (1987)

61. Y.M. Cho, Phys. Rev. Lett. 68, 3133 (1992)

62. Y.M. Cho, J.H. Yoon, Phys. Rev. D 47, 3465 (1993)

63. E. Babichev, Phys. Rev. D 74, 085004 (2006)

64. V. Kusmin, V. Rubakov, M. Shaposhnikov, Phys. Lett. B 155, 36 (1985)

65. L. Yaffe, Phys. Rev. D 40, 3463 (1989)

66. J. Gipson, H. Tze, Nucl. Phys. B 183, 524 (1981)

67. J. Gipson, Nucl. Phys. B 231, 365 (1984)

68. M. Clements, S. Tye, Phys. Rev. D 33, 1424 (1986)

69. Y.M. Cho, K. Kimm, J.H. Yoon, to be published 